Publ. RIMS, Kyoto Univ.

18 (1982), 57-99

\title{
On the Cauchy Problem of the Boltzmann Equation with a Soft Potential
}

By

\author{
Seiji UhAI* and Kiyoshi ASANo**
}

\section{Introduction}

The Cauchy problem to the non-linear Boltzmann equation is, [3].

$$
\left\{\begin{array}{l}
\frac{\partial f}{\partial t}=-\xi \cdot \Gamma_{x} f+Q[f, f], \quad(l, x, \xi) \in \mathbb{R}_{+} \times \mathbb{L}^{n} \therefore \mathbb{J n}^{n}, \\
\left.f\right|_{\ell=0}=f_{0} .
\end{array}\right.
$$

Here the unknown $f=f(t, x, \xi)$ is a function of $t \in \overline{\mathbb{R}}_{+}=[0, \infty)$, $x=\left(x_{1}, x_{2}, \cdots, x_{n}\right) \in \mathbb{R}^{n}$ and $\xi=\left(\xi_{1}, \xi_{2}, \cdots, \xi_{n}\right) \in \mathbb{R}^{n}$ while $\xi \cdot \nabla_{x}=\sum_{j=1}^{n} \xi_{j} \partial / \partial x_{j}$ and $Q$ is a bilinear symmetric operator given as

$$
\begin{aligned}
& Q[f, g]=\frac{1}{2} \int_{\mathbb{R}^{n} \times S^{n-1}} q\left(\left|\xi-\xi^{\prime}\right|, \theta\right) \\
& \quad \times\left\{f^{\prime}(\eta) g\left(\eta^{\prime}\right)+f\left(\eta^{\prime}\right) g(\eta)-f(\xi) g\left(\xi^{\prime}\right)-f\left(\xi^{\prime}\right) g(\hat{\xi})\right\} d \xi^{\prime} d \omega,
\end{aligned}
$$

where $f(\eta)=f(t, x, \eta)$ etc., $\eta=\xi-\left\langle\xi-\xi^{\prime}, \omega\right\rangle \omega, \quad \eta^{\prime}=\xi^{\prime}-\left\langle\xi-\xi^{\prime}, \omega\right\rangle(\omega$, $\omega \in S^{n-1}$ and $\cos \theta=\left\langle\xi-\xi^{\prime}, \omega\right\rangle /\left|\xi-\xi^{\prime}\right|,\langle\cdot, \cdot\rangle$ denoting the inner product in $\boldsymbol{R}^{n}$, and $q(v, \theta) \quad\left(v=\left|\xi-\xi^{\prime}\right|\right)$ is a given function defined on $\mathbb{R}_{+} \times(-\pi$, $\pi)$.

Physically (1.1) describes an evolution of a gas in the space $\mathbb{R}^{n}$ in terms of the (probability) density $\int=f(t, x, \xi)$ of gas particles at time $t$ in the space of position $x$ and relocity $\xi$. The operator $Q$ describes the binary collisions of gas particles and the function $q$ is determined by the corresponding interaction potential. For example we know $q(v, \theta)=d^{2} \mid\langle\xi$ $\left.-\xi^{\prime}, \omega\right\rangle \mid$ for the hard ball model [3], in which the gas particle is assumed

Communicated by S. Matsuura, December 19, 1980.

* Department of Applied Physics, Osaka City University, Sumiyoshi-ku, Osaka 558, Japan.

** Institute of Mathematics, Yoshida College, Kyoto University, Kyoto 606, Japan. 
to be a rigid sphere of diameter $d$, and

$$
q(v, \theta)=v^{\gamma} q_{0}, \quad r=\frac{s-5}{s-1}, \quad s>1
$$

for the potential of inverse power law $r^{-s}[6]$, where $r$ denotes the distance between two colliding gas particles. The function $q_{0}(\theta)$ is a nonnegative function only of $\theta$ and has a singularity at $\theta= \pm \pi / 2$ like $|\cos \theta|^{-\left(s^{+} 1\right) /(s-1)}$. But since $\eta=\xi, \eta^{\prime}=\xi^{\prime}$, at $\theta= \pm \pi / 2$, the quantity $\{\cdots\}$ in (1.2) is expected to vanish at these $\theta$, and it is customary to assume that $0 \leq q_{0}(\theta) \leq$ const. $|\cos \theta|$ (angular cutoff [6]). The inverse power law potential is said soft (resp. hard) if $s \leq 5$ (resp. $s \geq 5$ ). Clearly the hard ball model is a limit case $s \rightarrow \infty$ of (1.3) with angular cutoff.

The aim of the present paper is to show the existence of solutions to (1.1) in the large in time under the following assumption on $q(v, \theta)$.

[Q] (0) $q(v, \theta)$ is a nonnegative measureable function on $\boldsymbol{R}_{+}$ $\times(-\pi, \pi)$.

(i) $0 \leq q(v, \theta) \leq q_{0}|\cos \theta|\left(v+v^{-\delta_{0}}\right)$

with some constants $q_{0} \geq 0$ and $\delta_{0} \in[0,1)$.

(ii) $\quad q_{1}(1+v)^{-\delta_{1}} \leq \int_{s^{n-1}} q(v, \theta) d \omega \leq q_{2} v^{-\delta}$

with some constants $q_{2} \geq q_{1}>0$ and $0 \leq \delta \leq \delta_{1}<1$.

Clearly (1.3) with angular cutoff satisfies [Q] (i) if $s>3$ and [Q] (ii) if $s \leq 5$. If we allow $q_{1}=0$, then the assumption [Q] (i) + (ii) is the same as that of cutoff soft potential proposed by Grad [6]. (The necessity of the assumption $q_{1}>0$ will be made clear in Remark 4.1 in the below.)

The initial value problem (1.1) and related initial-boundary value problems have been studied extensively on the existence of solutions in the large in time ([1], [5], [9], [12], [13], [14], [15]). All of these works, however, have been made under the assumption of cutoff hard potential in the sense of Grad [6], given also by [Q] but with the modification $-1<\delta_{2} \leq \delta \leq 0$ in [Q] (ii), and satisfied by the hard ball model and by (1.3) if $s \geq 5$ and if the angular cutoff is assumed.

A remarkable distinction is found on the existence of local (in time) solutions. Roughly speaking, (1.1) admits local solutions for arbitrary 
initials $f_{0}$ in the case of cutoff soft potentials (Theorem 3.2 below), While for cutoff hard potential such solutions are known at the present stage only for those $f_{0}$ near Maxwellians (for definition see $\S 3$ ), which are eventually in the large in time [13]. Thus the cutoff soft potentials enjoy a better situation in this respect.

However a considerable complexity arises when constructing the solutions in the large in time. Our method of proof is the same as that previously developed for the case of cutoff hard potentials, which is based on an extensive use of asymptotic behaviors of a linear semigroup $e^{t B}$ of the so-call linearized Boltzmann operator $B$ obtained upon the linearization of (1.1) (see $\S 3$ ). In order to establish decay estimates of $e^{t B}$ as $t \rightarrow \infty$ which are nice enough to assure the nonlinear problem (1.1) to have solutions in the large in time, the behaviors of the resolvent $(\lambda-B)^{-1}$ should be carefully studied near the imaginary axis $\operatorname{Re} \lambda=0$.

For cutoff hard potentials, the spectrum $\sigma(B)$ of $B$ has a fairly simple structure near $\operatorname{Re} \lambda=0$ : either a finite number of discrete eigenvalues ([5], [12], [13]) or a continuous spectrum consisting of a finite number of smooth curves emerging from $\lambda=0$ ([1], [4], [9], [14], [15]):

On the other hand, $\sigma(B)$ for cutoff soft potentials contains a left neighborhood of the imaginary axis (Remark 7.3). In order to establish estimates of the resolvent $(\lambda-B)^{-1}$ near $\operatorname{Re} \lambda=0$, therefore, we are forced to use its $C^{\infty}$-extension into $\operatorname{Re} \lambda \leq 0$, which were not needed in the case of cutoff hard potentials.

The plan of the present paper is as follows. In Section 2 we introduce some function spaces in which our problem is to be studied, and we also summarize the notations for linear operators, both of which will be used without further references in what follows. The local existence of solutions is proved in Section 3 while the solutions in the large in time are constructed in Section 9. The intermediate Sections 4 to 8 are devoted exclusively to the study of linear operators associated with $B$ to establish asymptotic behaviors of $e^{t B}$ which play an essential role in Section 9. Thus these sections have a preliminary character to Section 9 , but form a core of the present paper.

After the conpletion of the present work we learned that Caflisch [3] also solved (1.1) for cutoff soft potentials, but imposing a periodic 
boundary condition in $x$. His method of proof is quite different from ours on the estimation of $e^{t B}$, and is effective to the case of periodic boundary condition with rather restricted initial values. The method presented in this paper allows a mild condition on initial values and is applicable to the periodic boundary value problem. Moreover the estimates derived here can be combined with the method developed in [1] [13] to solve exterior problems to (1.1) with soft potentials in the large in time. This is a subject in a forthcoming paper.

Remark. Recently we have obtained almost the same results for the Boltzmann equation with an angular cutoff soft potential of exponent $s \in(7 / 3,3]$, that is, $1 \leq \delta_{0}=\delta_{1}<2$ in the assumption [Q]. The details will be discussed in another place.

\section{$\S 2$. Function Spaces and Notations for Linear Operators}

Let $\Omega \subset \boldsymbol{R}^{n}$ be a domain. We will write $\Omega_{y}$ when it is necessary to emphasize that the symbol for the generic point of $\Omega$ is specified as $y$. The closure of $\Omega$ will be denoted as $\bar{\Omega}$.

For $1 \leq p \leq \infty, L^{p}(\Omega)$ will denote the usual Lebesgue spaces on $\Omega$. It is a Banach space with the well-known definition of norm (denoted as $\left.\|\cdot\|_{L^{p}(\Omega)}\right)$. By definition $L^{p}\left(\Omega_{y}\right)$ is the class of functions $u=u(y)$ $\in L^{p}(\Omega)$ whose variable is $y . \quad L_{l o c}^{p}(\Omega)$ denotes the class of locally $L^{p}(\Omega)$ functions. Let $\beta \in \boldsymbol{R}$ and define

$$
L_{\beta}^{p}\left(\boldsymbol{R}^{n}\right)=\left\{u=u(\xi) ;(1+|\xi|)^{\beta} u(\xi) \in L^{p}\left(\boldsymbol{R}^{n}\right)\right\},
$$

which will be abbreviated sometimes as $L_{\beta}^{p}$, and in particular as $L^{p}$ when $\beta=0$. This is a Banach space with the natural norm

$$
\|u\|_{L_{\beta}^{p}}=\left\|(1+|\xi|)^{\beta} u(\xi)\right\|_{L^{p}\left(\boldsymbol{R}^{n}\right)}
$$

and coincides with $L^{p}\left(\boldsymbol{R}^{n}\right)$ when $\beta=0$.

Let $l=0,1,2, \cdots, \infty . \quad C^{l}(\Omega), C_{0}^{l}(\Omega)$ and $C^{0}(\bar{\Omega})$ have usual meanings and we put $\mathscr{B}^{0}(\bar{\Omega})=C^{0}(\bar{\Omega}) \cap L^{\infty}(\Omega)$. For $l \geq 1, C^{l}(\bar{\Omega})\left(\right.$ resp. $\mathscr{B}^{l}(\bar{\Omega})$ ) denotes the class of $C^{0}(\bar{\Omega})$ (resp. $\mathscr{B}^{0}(\bar{\Omega})$ )-functions whose derivatives up to order $l$ inclusive exist in $\Omega$ and have $C^{0}(\bar{\Omega})$ (resp. $\mathscr{B}^{0}(\bar{\Omega})$ ) -extension in $\bar{\Omega}$. Clearly $C^{l}(\bar{\Omega})=\mathscr{B}^{l}(\bar{\Omega})$ if $\Omega$ is bounded. 
For $l \in \mathbb{R}, H^{l}\left(\mathbb{R}^{n}\right)$ denotes the usual $L^{2}$-sense Sobolev space on $\mathbb{R}^{n}$ of order $l$;

$$
H^{l}\left(\mathbb{R}^{u}\right)=\left\{u \in L_{l o c}^{2}\left(\mathbb{R}^{n}\right) ; \hat{u} \in L_{l}^{2}\left(\mathbb{R}^{n}\right)\right\},
$$

where $\widehat{u}=\widehat{u}(k)$ is the Fourier transform of $u=u(x)$;

$$
\widehat{u}(k)=(2 \pi)^{-n / 2} \int_{\mathbb{R}^{n}} e^{-i\langle x, k\rangle} u(x) d x, \quad k \in \mathbb{P}^{n} .
$$

It is well-known that $I H^{l}\left(\mathbb{R}^{n}\right)$ is a Banach (more precisely Hilbert) space with the norm

$$
\|u\|_{\boldsymbol{H}^{\imath}\left(\mathbb{R}_{x}^{n}\right)}=\|\hat{u}\|_{L_{l}^{2}\left(\mathbb{R}_{k}^{n}\right)},
$$

and further that it is a Banach algebra if $l>n / 2$.

Let $Y(\Omega)$ be any one of the function spaces introduced so far and let $X$ be a Banach space with the norm $\|\cdot\|_{X}$. Then $Y(\Omega ; X)$ will denote the class of $Y(\Omega)$-functions defined on $\Omega$ with values in $X$. If $Y(\Omega)$ is a Banach space, then so is $Y(\Omega ; X)$ with the norm

$$
\|u\|_{Y(\Omega ; X)}=\|\| u(\cdot)\left\|_{X}\right\|_{Y(\Omega)} .
$$

The space $Y(\bar{Q} ; X)$ is similarly defined. For example we shall need the space

$$
H_{l, \beta, p}=L_{\beta}^{p}\left(\mathbb{R}_{\xi}^{n} ; H^{l}\left(\mathbb{R}_{x}^{n}\right)\right), \quad l, \beta \in \mathbb{R}, \quad 1 \leq p \leq \infty
$$

and we pur

$$
\|u\|_{l, \beta, p}=\|\| u(x, \xi)\left\|_{H^{l}\left(\boldsymbol{R}_{x}^{n}\right)}\right\|_{L_{\beta}^{p}\left(\boldsymbol{R}_{\xi}^{n}\right)} .
$$

The suffix $p$ will be dropped in the special case $p=\infty$. Thus

$$
H_{l, \beta}=H_{l, \beta, \infty}=L_{\beta}^{\infty}\left(\mathbb{R}_{\xi}^{n} ; H^{l}\left(\mathbb{R}_{x}^{n}\right)\right), \quad\|u\|_{l, \beta}=\|u\|_{l, \beta, \infty},
$$

which is obviously a Banach algebra if $l>n / 2$ and $\beta \geq 0$.

The function space in which our problem is to be solved is the space

$$
\dot{H}_{l, \beta}=\text { closure of } \bigcap_{l^{\prime}, \beta^{\prime} \in \mathbb{R}^{n}} H_{l^{\prime}, \beta^{\prime}} \text { in } H_{l, \beta} \text {. }
$$

This is not empty since $C_{0}^{\infty}\left(\mathbb{R}_{x}^{n} \times \mathbb{R}_{\xi}^{n}\right)$ is contained (but not dense) in $\dot{H}_{l, \beta}$. Further it is not difficult to see that $u \in \dot{H}_{l, \beta}$ if and only if $u \in H_{l, \beta}$ with

$$
\lim _{R \rightarrow \infty}\left\|\chi_{R}(\xi) u\right\|_{l, \beta}=\lim _{R \rightarrow \infty}\|\| \chi_{R}(k) \hat{u}(k, \xi)\left\|_{L_{i}^{2}\left(\boldsymbol{R}_{k}^{n}\right)}\right\|_{L_{\beta}^{\infty}\left(\boldsymbol{R}_{\xi}^{n}\right)}=0
$$

where $\chi_{k}(y)$ is defined as 


$$
\chi_{R}\left(y^{\prime}\right)=1 \text { for }|y| \geq R, \quad=0 \text { for }|y|<R .
$$

Thus $\dot{H}_{l, \beta}$ is itself a Banach space with the norm $\|\cdot\|_{l, \beta}$ of (2.8) as a proper subset of $H_{l, \beta}$. Note that

$$
\dot{H}_{l, \beta} \subset \dot{H}_{l^{\prime}, \beta}, \quad\|u\|_{l^{\prime}, \beta} \leq\|u\|_{l, \beta}
$$

for any $l^{\prime} \leq l$ and $\beta^{\prime} \leq \beta$.

We also need the space

$$
L^{p, 2}=L^{2}\left(\boldsymbol{R}_{\xi}^{n}: L^{p}\left(\boldsymbol{R}_{x}^{n}\right)\right),
$$

whose norm will be denoted as $\|\cdot\|_{L^{p, 2}}$.

Now we shall summarize some notations on linear operators. Let $X, Y$ be Banach spaces. We denote by $\boldsymbol{B}(X, Y)$ the class of linear bounded operators defined on $X$ with range in $Y$. and by $C(X, Y)$ its subset of compact operators. The norm of $A \in \boldsymbol{B}(X, Y)$ will be denoted as $\|A\|_{\boldsymbol{B}(X, Y)}$. Endowed with this norm, both $\boldsymbol{B}(X, Y)$ and $\boldsymbol{C}(X, Y)$ are Banach spaces. We will write $\boldsymbol{B}(X)$ for $\boldsymbol{B}(X, X)$ and $\boldsymbol{C}(X)$ for $C(X, X)$.

Let $A$ be a linear (not necessary bounded) operator defined in $X$ with range also in $X$. The domain of $A$ will be denoted as $D(A)$. By definition $D(A)=X$ if $A \in \boldsymbol{B}(X)$. Let $\rho(A)$ and $\sigma(A)$ denote respectively the resolvent set and the spectrum of $A$, and $(\lambda-A)^{-1}$ $=(\lambda I-A)^{-1}$ its resolvent where $I$ is the identity and $\lambda \in \rho(A)$. The symbols $\sigma_{p}(A), \sigma_{d}(A)$ and $\sigma_{e}(A)$ will stand respectively for the set of eigenvalues (point spectrum), the set of discrete eigenvalues, and the essential spectrum (in the sense of $[8, \mathrm{p} .243]$ ) of $A$. The adjoint to $A$ if it exist is denoted as $A^{*}$.

Finally we shall agree with the following convention. Let $A$ be a formally defined operator. Then $A$ may happen to have various realizations in various spaces. In fact we shall often need in the sequel to consider $A$ in a variety of spaces. For simplification of notation, therefore, we will use one and the same symbol $A$ for all of such realizations. No confusions will arise. Thus, for example, the statement $A \in \boldsymbol{B}(X, Y)$ will be always understood to mean that $A$ has a (unique) realization belonging to $\boldsymbol{B}(X, Y)$. 


\section{§3. Existence of Local Solutions}

It is well-known [3] that $Q[g, g]=0$ if $g(\xi)=\alpha \exp \left\{-\gamma|\xi-\beta|^{2}\right\}$ whatever the constants $\alpha \in \boldsymbol{R}, \beta \in \mathbb{R}^{n}, \gamma>0$ may be. Consequently $g=g(\xi)$ satisfies the Boltzmann equation in (1.1). If $\alpha>0, g(\xi)$ is called Maxwellian and describes an equilibrium state of a gas.

In the present paper we shall seek a solution to (1.1) which is near a Maxwellian $g(\xi)$. Without loss of generality we may choose $\alpha=1, \beta=0, \gamma=1 / 2$; otherwise we can reduce to this case by a suitable change of variables. Throughout this paper, therefore, $g$ will be understood the function $g(\xi)=\exp \left\{-|\xi|^{2} / 2\right\}$.

Putting $f=g+g^{1 / 2} u$, we rewrite (1.1) in terms of the new unknown $u=u(t, x, \xi)$ as

$$
\left\{\begin{array}{l}
\frac{\partial u}{\partial t}=-\hat{s} \cdot \nabla_{x} u+L u+\Gamma[u, u] \\
\left.u\right|_{t=0}=u_{0}
\end{array}\right.
$$

where we have put

$$
\begin{aligned}
& \Gamma[u, v]=g^{-1 / 2} Q\left[g^{1 / 2} u, g^{1 / 2} v\right], \\
& L u=2 g^{-1 / 2} Q\left[g, g^{1 / 2} u\right]=2 \Gamma\left[g^{1 / 2}, u\right] .
\end{aligned}
$$

The operator $\Gamma$ is bilinear symmetric while $L$ is linear, both acting only on the variable $\xi$.

Define

$$
\text { (3.4) } \begin{aligned}
\Gamma_{1}[u, v] & =\frac{1}{2} \int_{\mathbb{R}^{n} \times S^{n-1}} q\left(\left|\xi-\xi^{\prime}\right|, \theta\right) g^{1 / 2}\left(\xi^{\prime}\right) u(\xi) v\left(\xi^{\prime}\right) d \xi^{\prime} d \omega, \\
\Gamma_{2}[u, v] & =\frac{1}{2} \int_{\mathbb{R}^{n} \times S^{n-1}} q\left(\left|\xi-\xi^{\prime}\right|, \theta\right) g^{1 / 2}\left(\xi^{\prime}\right) u(\eta) v\left(\eta^{\prime}\right) d \xi^{\prime} d \omega,
\end{aligned}
$$

where the notations are the same as in (1.2). It is easy to see that

$$
\Gamma[u, v]=\Gamma_{2}[u, v]+\Gamma_{2}[v, u]-\Gamma_{1}[u, v]-\Gamma_{1}[v, u] .
$$

Let $L_{\beta}^{\infty}\left(\boldsymbol{R}_{\xi}^{n}\right)$ be that defined in (2.1) with $p=\infty$ and write its norm (2.2) simply as $\|\cdot\|_{\beta}$.

Lemma 3. 1. Assume [Q] (0) and (ii). There is a constant 
$c_{0} \geq 0$ such that for any $\beta \geq 0$ and $u, v \in L_{\beta}^{\infty}\left(\boldsymbol{R}_{\xi}^{n}\right)$,

$$
\left\|\Gamma_{j}[u, v]\right\|_{\beta+\delta} \leq \mathrm{c}_{0}\|u\|_{\beta}\|v\|_{\beta}, \quad j=1,2,
$$

where $\delta$ is the constant in [Q] (ii).

Proof. Put

$$
\sigma(\xi)=\int_{R^{n} \times S^{n-1}} q\left(\left|\xi-\xi^{\prime}\right|, \theta\right) g^{1 / 2}\left(\xi^{\prime}\right) d \xi^{\prime} d \omega
$$

Owing to [Q] (ii), there is a constant $\gamma_{0} \geq 0$ such that

$$
0 \leq \sigma(\xi) \leq \gamma_{0}(1+|\xi|)^{-\delta}, \quad \xi \in \boldsymbol{R}^{n} .
$$

Then the lemma follows if we prove

$$
\left|\Gamma_{j}[u, v](\xi)\right| \leq(1+|\xi|)^{-\beta} \sigma(\xi)\|u\|_{\beta}\|v\|_{\beta} .
$$

For $j=1$, this is a simple consequence of the definitions of $\Gamma_{1}$ and $\|\cdot\|_{\beta}$, and to prove the case $j=2$ it suffices to note that $|\eta|^{2}+\left|\eta^{\prime}\right|^{2}=|\xi|^{2}+\left|\xi^{\prime}\right|^{2}$ so that

$$
\begin{array}{r}
(1+|\eta|)\left(1+\left|\eta^{\prime}\right|\right) \geq 1+|\eta|+\left|\eta^{\prime}\right| \\
\geq 1+\sqrt{|\eta|^{2}+\left|\eta^{\prime}\right|^{2}} \geq 1+|\xi| .
\end{array}
$$

This lemma suggests that a suitable function space in which (3.1) is to be solved is the Banach space $H_{l, \beta}$ defined by (2.8). However more suitable is $\dot{H}_{l, \beta}$ of (2.9) as will be seen in Remark 3.1 in the below.

Since $\Gamma$ and $L$ are operators acting only on the variable $\xi$, they can be considered in the space $\dot{H}_{l, \beta}$ and $H_{l, \beta}$ as well.

Lemma 3. 2. Assume [Q] (0) and (ii).

(i) Let $l>n / 2$ and $\beta \geq 0$. Then $\Gamma$ is a bounded bilinear symmetric operator from $\dot{H}_{l, \beta} \times \dot{H}_{l, \beta}$ into $\dot{H}_{l, \beta+\delta}$.

(ii) For any $l \in \boldsymbol{R}$ and $\beta \geq 0, L$ is a bounded linear operator from $\dot{H}_{l, \beta}$ into $\dot{H}_{l, \beta+\delta}$, that is, $L \in \boldsymbol{B}\left(\dot{H}_{l, \beta}, \dot{H}_{l, \beta+\delta}\right)$.

Proof. (i) Let $u \in H_{l, \beta}$ and put $\iota_{0}(\xi)=\|u(\cdot, \xi)\|_{H l\left(\boldsymbol{R}_{x}^{n}\right)}$ and similarly for $v \in H_{l, \beta}$. Then $u_{0}, v_{0} \in L_{\beta}^{\infty}$. Let $l>n / 2$. Since $H^{l}\left(\boldsymbol{R}^{n}\right)$ is a 
Banach algebra and since $q(v, \theta) \geqq 0$, we see that

$$
\left\|\Gamma_{j}[u, v](\cdot, \xi)\right\|_{H^{l}\left(\boldsymbol{R}_{x}^{n}\right)} \leq C \Gamma_{j}\left[u_{0}, v_{0}\right](\xi), \quad j=1,2,
$$

the constant $C \geq 0$ depending only on $n$ and $l$. By Lemma 3.1 and (3.5), we then get for any $l>n / 2$ and $\beta \geq 0$,

$$
\|\Gamma[u, v]\|_{l, \beta+\delta} \leq C \gamma_{0}\|u\|_{l, \beta}\|r\|_{l, \beta} .
$$

This proves the assertion (i) of the lemmat with $I I$ in place of $\dot{H}$. To complete the proof, it suffices to show that $\Gamma[u, v] \in \dot{H}_{l, \beta+\delta}$ whenever $u, v \in \dot{H}_{l, \beta}$. So let $u, v \in \dot{H}_{l, \beta}$. By the definition (2.9), there exist sequences $\left\{u_{n}\right\}$ and $\left\{v_{n}\right\}$ in $\bigcap_{l, \beta} H_{l, \beta}$, which converge to $u$ and $v$ strongly in $H_{l, \beta}$. Then it follows from (3.6) not only that $I^{\top}\left[u_{n}, v_{n}\right] \in \bigcap_{l, \beta} H_{l, \beta}$ for all $n$, but also, by virtue of the bilinearity of $\Gamma$, that $\Gamma\left[u_{n}, v_{n}\right]$ converges to $\Gamma[u, v]$ strongly in $H_{l, \beta+\delta}$, showing that $\Gamma[u, v] \in \dot{H}_{l, \beta+\delta}$.

(ii) Since $g(\xi)$ is independent of $x$, we have

$$
\left\|\Gamma_{j}\left[g^{1 / 2}, u\right](\cdot \xi)\right\|_{H^{l}(\boldsymbol{R})_{x}^{n}} \leq \Gamma_{j}\left[g^{1 / 2}, u_{0}\right](\xi), \quad j=1,2,
$$

where $l \in \mathbb{R}$ and $\beta \geq 0$. Clearly $g^{1 / 2}$ and $"$ can be interchanged. By Lemma 3.1 again and by (3.5) applied to (3.3), we get

$$
\|I, u\|_{l, \beta \cdot \delta} \leq \gamma_{\mathrm{u}}\|g\|_{\beta}\|u\|_{l, \beta} .
$$

The rest of the proof is similar to that of (i) and will be omitted.

Since $\delta \geq 0$ and since $\Gamma$ is bilinear, Lemma 3.2 and (2.12) lead to

Corollary 3.1. (i) Let $l>n / 2$ and $\beta \geq 0$. Then the quadratic operator $\Gamma[u, u]$ is a $C^{\infty}$-map from $\dot{H}_{l, \beta}$ into itself.

(ii) $L$ is a linear bounded operator on $\dot{H}_{l, \beta}$ for any $l \in \mathbb{R}$ and $\beta \geq 0$, namely $L \in \mathbb{B}\left(\dot{H}_{l, \beta}\right)$.

Let us return to (3.1). The linearized Boltzmann operator $B$ is the linear operator appearing in the right side of (3.1);

$$
(B u)(x, \xi)=-\xi \cdot \nabla_{x} u(x, \xi)+(L u)(x, \xi) .
$$

We shall study it in the space $\dot{H}_{l, \beta}$ with the domain

$$
D(B)=\left\{u \in \dot{H}_{l, \beta} ; \xi \cdot \nabla_{x} u \in \dot{H}_{l, \beta}\right\},
$$


where $\xi \cdot \nabla_{x}$ is, of course, in the distribution sense. Note that this choice is maximal in $\dot{H}_{l, \beta}$ in virtue of Corollary 3.1 (ii), and that $\dot{H}_{l+1, \beta+1}$ $\subset D(B)$,

Theorem 3. 1. Assume [Q] (0) and (ii), and let $l \in \boldsymbol{R}, \beta \geq 0$. Then $B$ is a generator of $C_{0}$-semigroup $e^{t B}$ on $\dot{H}_{l, \beta}$.

Proof. Define the operator $A_{0}$ as

$$
A_{0} u=-\xi \cdot \nabla_{x} u, \quad D\left(A_{0}\right)=D(B) .
$$

Then $B=A_{0}+L$, and since $L$ is a bounded perturbation to $A_{0}$ (Corollary 3.1 (ii)), it suffices to prove that $A_{0}$ is a generator, or equivalently that $A_{0}$ satisfies the conditions of the Hille-Yoshida theorem. Clearly $\bigcap_{l, \beta} H_{l, \beta}$ $\subset D\left(A_{0}\right)$, so $D\left(A_{0}\right)$ is dense in $\dot{H}_{l, \beta}$ by (2.9). Further the Fourier transformation with respect to $x$ gives

$$
\left(A_{0} u\right)^{\wedge}(k, \xi)=-i\langle k, \xi\rangle \widehat{u}(k, \xi)
$$

in the distribution sense, and thereby we get formally,

$$
\left(\left(\lambda I-A_{0}\right)^{-1} u\right)^{\wedge}(k, \xi)=(\lambda+i\langle k, \xi\rangle)^{-1} \hat{u}(k, \xi),
$$

for $\lambda \in C$. But this is exact in $\dot{H}_{l, \beta}$ if $\operatorname{Re} \lambda \neq 0$, as is seen by the aid of (2.10). Thus $\rho\left(A_{0}\right)=\boldsymbol{C} \backslash i \boldsymbol{R}$ and

$$
\left\|\left(\lambda I-A_{0}\right)^{-1}\right\|_{B\left(\dot{H}_{l, \beta)}\right.} \leq|\operatorname{Re} \lambda|^{-1} .
$$

This proves also that $A_{0}$ is closed. Now the Hille-Yoshida theorem assures that $A_{0}$ generates a contraction group on $\dot{H}_{l, \beta}$, completing the proof of the theorem.

Note that (3.10) implies also

$$
\left(e^{t A_{0}} u\right)^{\wedge}(k, \xi)=e^{-i t\langle k, \xi\rangle} \widehat{u}(k, \xi) .
$$

Remark 3.1. In the space $H_{l, \beta}, A_{0}$ is not densely defined even if endowed with a maximal domain, and $e^{t A_{0}}$ of (3.11) is not strongly continuous in $t$. Thus $A_{0}$ cannot be a generator in $H_{l, \beta}$. The situation is the same for $B$, and this is the reason why $\dot{H}_{l, \beta}$ is preferable to $H_{l, \beta}$. 
We now consider (3.1) as an evolution equation in $\dot{H}_{l, \beta}$;

$$
\left\{\begin{array}{l}
\frac{d u(t)}{d t}=B u(t)+\Gamma[u(t), u(t)], t>0, \\
u(+0)=u_{0} .
\end{array}\right.
$$

Here $l>n / 2$ and $\beta \geq 0$, and $d u(t) / d t$ is, of course, a strong derivative of $u(t)$ in $t$ in $\dot{H}_{l, \beta}$. We shall agree to say that $u=u(t)$ is a solution to (3.12) in $\dot{H}_{l, \beta}$ in the interval $[0, T)$ with some $T>0$, if $u \in C^{1}([0, T)$; $\left.\dot{H}_{l, \beta}\right)$ and $u(t) \in D(B)$ for all $t \in[0, T)$ and if $u$ satisfies (3.12).

Let $u=u(t)$ be such a solution. Then it is obvious that $u$ satisfies the integral equation

$$
u(t)=e^{t B} u_{0}+\int_{0}^{t} e^{(t-s) B} \Gamma[u(s), u(s)] d s
$$

for $t \in[0, T)$. Conversely if $u(t) \in C^{0}\left([0, T) ; \dot{H}_{l, \beta}\right)$ is a solution to (3.13), then it is also a solution to (3.12) in the above sense so far as $u_{0} \in D(B)$. This follows essentially from Corollary 3.1 (i). And (3.13) can be solved by the classical contraction mapping principle in virtue of Theorem 3.1 and Corollary 3.1 (i). Thus we conclude the

Theorem 3.2 (Existence of local solutions). Suppose that [Q] (0) and (ii) be fulfilled, and let $l>n / 2$ and $\beta \geq 0$.

(i) For any $u_{0} \in \dot{H}_{l, \beta}$ there exists a positive constant $T$ and (3.13) has a unique solution $u=u(t)$ in $C^{0}\left([0, T) ; \dot{H}_{l, \beta}\right)$.

(ii) If, in addition, $u_{0} \in D(B)$, then this $u$ is also a unique solution to $(3.12)$ in $\dot{H}_{l, \beta}$ in $[0, T)$.

Remark 3.2. The assumption $q_{1}>0$ in [Q] (ii) was not used in the proof of Lemma 3.1. Thus Theorem 3.2 remains valid even if $q_{1}=0$. This assumption is essential, however, for our construction of solutions in the large in time (see Remark 5.1).

Remark 3.3. No theorems of the type of Theorem 3.2 are not yet known for the case of cutoff hard potentials. In fact if $\delta<0$ in [Q] (ii), Corollary 3.1 is no longer true though Lemmas 3.1 and 3.2 remain valid. Thus the proof of Theorem 3.2 does not apply to the 
hard ball model and the inverse power potentials (1.3) with $s>5$. The local solutions shown in [7] are only for small $u_{0}$ and can be continued eventually in the large in time [9], [13], [14].

\section{§4. Collision Operators}

In order to construct the solutions to (3.12) in the large in time, we need to establish nice decay estimates of $e^{t B}$ for large $t$, and to this end we shall first study the operator $L$ of (3.3) in some details.

Let $\Gamma_{j}(j=1,2)$ be as in $(3.4)$ and put

$$
\Lambda \imath=\Gamma_{1}\left[u, g^{1 / 2}\right] \text {, }
$$

$$
K u=L u+\Lambda u=\Gamma_{2}\left[u, g^{1 / 2}\right]+\Gamma_{2}\left[g^{1 / 2}, u\right]-\Gamma_{1}\left[g^{1 / 2}, u\right]
$$

where $g(\xi)=\exp \left\{-|\xi|^{2} / 2\right\}$. The aim of this section is to study $A, K$ and $L$. We shall do this under the assumption [Q] of Section 1, with the restriction

$$
\delta=\delta_{0}=\delta_{1}
$$

This additional assumption is not essential, and is made only to shorten our arguments.

As is easily seen, $\Lambda$ is a multiplication operator $(\Lambda u)(\xi)=\nu(\xi) u(\xi)$ by the function

$$
\nu(\xi)=\frac{1}{2} \int_{\boldsymbol{R}^{n \times S^{n-1}}} q\left(\left|\xi-\xi^{\prime}\right|, \theta\right) g^{1 / 2}\left(\xi^{\prime}\right) d \omega d \xi^{\prime},
$$

which, under the assumption [Q] (0), is measurable on $\boldsymbol{R}^{n}$, and in view of [Q] (ii),

$$
\nu_{1}(1+|\xi|)^{-\delta} \leq \nu(\xi) \leq \nu_{2}(1+|\xi|)^{-\delta}
$$

with some constants $\nu_{1}, \nu_{2}>0$. The fact that $\nu_{1}>0$ assures that for all $\alpha \in \boldsymbol{R}$

$$
0 \leq \nu(\xi)^{\alpha} \leq \nu_{\alpha}(1+|\xi|)^{-\alpha \delta}
$$

with $\nu_{\alpha}<+\infty$, so that we can define (formally) the fractional power $A^{\alpha}$ even for $\alpha<0$ as

$$
\left(\Lambda^{\alpha} u\right)(\xi)=\nu(\xi)^{\alpha} u(\xi)
$$


Remark 4.1. The validity of (4.3) for $\alpha<0$ is essential for our construction of global solutions and follows from the assumption $q_{1}>0$ in [Q] (ii).

Lemma 4. 1. (i) Let $\alpha, \beta, \gamma \in \mathbb{R}$ be such that $\gamma \leq \beta+\alpha \delta$, and let $l \in \mathbb{R}$ and $p \in[1, \infty]$. Then $\Lambda^{\alpha} \in \mathbb{B}(X, Y)$ for any one of the following pairs of spaces $(X, Y)$.
(a) $\left(L_{\beta}^{p}, L_{r}^{p}\right)$,
(b) $\left(\dot{H}_{l . A}, \dot{H}_{l, r}\right)$,
(c) $\left(H_{l, \beta, p}, H_{l, r, p}\right)$.

(ii) For any $\beta \in \boldsymbol{R}, \Lambda$ is a bounded nonnegative selfadjoint operator on the Hilbert space $L_{\beta}^{2}$, with $\sigma(\Lambda)=\sigma_{e}(\Lambda)=\left[0, \nu_{0}\right]$ where $\nu_{0}$ $=\sup _{\xi} \nu(\xi)$.

Proof. Evident from (4.3).

Let us now study the operator $K$ of (4.2). It is easy to see that $\Gamma_{1}\left[g^{1 / 2}, u\right]$ is an integral operator

$$
\Gamma_{1}\left[g^{1 / 2}, u\right](\hat{\xi})=\int_{\mathbb{R}^{n}} K_{1}\left(\hat{\xi}, \xi^{\prime}\right) u\left(\xi^{\prime}\right) d \hat{\xi}^{\prime}
$$

induced by the kernel

$$
K_{1}\left(\xi, \xi^{\prime}\right)=\frac{1}{2} g^{1 / 2}(\xi) g^{1 / 2}\left(\hat{\xi}^{\prime}\right) \int_{S^{n-1}} q\left(\left|\dot{\xi}-\hat{\xi}^{\prime}\right|, \theta\right) d \omega
$$

which is nonnegative measurable on $\mathbb{R}_{\xi}^{n} \times \mathbb{R}_{\xi}^{n}$ by $[Q](0)$ and symmetric with respect to $\xi$ and $\xi^{\prime}$. Further [Q] (ii) assures

$$
0 \leq K_{1}\left(\xi, \xi^{\prime}\right) \leq \frac{1}{2} q_{2}\left|\xi-\xi^{\prime}\right|^{-\delta} \exp \left\{-\left(|\xi|^{2}+\left|\xi^{\prime}\right|^{2}\right) / 4\right\}
$$

Also the operator $\Gamma_{2}\left[g^{1 / 2}, u\right]+\Gamma_{2}\left[u, g^{1 / 2}\right]$ is an integral operator induced by a kernel $K_{2}\left(\xi, \xi^{\prime}\right)$ which is real, measurable and symmetric on $\mathbb{R}^{n}$ 冫 $\mathbb{R}^{n}$ by [Q] (0), and [Q] (i) implies that

$$
\begin{aligned}
0 \leq & K_{2}\left(\xi, \xi^{\prime}\right) \leq k_{2}\left(\left|\xi-\xi^{\prime}\right|^{-1}+\left|\xi-\xi^{\prime}\right|^{-(n-2)}\right) \\
& \times \exp \left[-\left\{\left|\xi-\xi^{\prime}\right|^{2}-\left(|\xi|^{2}-\left|\xi^{\prime}\right|^{2}\right)^{2} /\left|\xi-\xi^{\prime}\right|^{2}\right\} / 8\right]
\end{aligned}
$$

with some constant $k_{2} \geq 0$. This has been proved in [6] for $n=3$ and in [11] for arbitrary $n \geq 2$, by using too complicated change of variables 
to reproduce their proofs here.

Consequently $K$ is also an integral operator

$$
(K u)(\xi)=\int_{\boldsymbol{R}^{n}} K\left(\xi, \xi^{\prime}\right) u\left(\xi^{\prime}\right) d \xi^{\prime}
$$

and the kernel $K\left(\xi, \xi^{\prime}\right)=K_{2}\left(\xi, \xi^{\prime}\right)-K_{1}\left(\xi, \xi^{\prime}\right)$ is real, measurable and symmetric on $\boldsymbol{R}^{n} \times \boldsymbol{R}^{n}$. Moreover using (4.5) and (4.6), we can easily get

$$
\int_{\boldsymbol{R}^{n}}\left|K\left(\xi, \xi^{\prime}\right)\right|^{p}(1+|\xi|)^{-\beta} d \xi^{\prime} \leq \kappa_{p, \beta}(1+|\xi|)^{-\beta-1},
$$

with a constant $\kappa_{p, \beta} \geq 0$, for any $\beta \geq 0$ and $0<p<n /(n-2)$ if $n \geq 3$ or $0<p<2$ if $n=2$.

Remark 4.2. No estimates other than (4.8) are needed in the sequel. Thus the assumption [Q] (i) is required only for (4.6) to be true.

Lemma 4.2. (i) Let $\beta, \gamma \in \boldsymbol{R}$ be such that $\gamma \leq \beta+1$ and let $l \in \boldsymbol{R}$ and $p \in(1, \infty)$. Then $K \in \boldsymbol{B}(X, Y)$ for any one of the following pairs $(X, Y)$.

(a) $\left(L_{\beta}^{p}, L_{r}^{p}\right), \quad$ (b) $\left(H_{l, \beta, p}, H_{l, r, p}\right)$

(c) $\left(\dot{H}_{l, \beta}, \dot{H}_{l, r}\right)$, where $\beta \geq 0$.

(d) $\left(H_{l, \beta, p}, \dot{H}_{l, r}\right)$, where

$\beta \geq 0, \gamma \leq \beta+1-1 / p, p>n / 2$ for $n \geq 3$ or $p>2$ for $n=2$.

(e) $\left(H_{l, \beta, p_{1}}, H_{l, r, p_{2}}\right)$ where $\beta, \gamma, p$ are as in (d), and $1<p_{1} \leq p, 1 / p_{1}-1 / p_{2} \leq 1 / p$.

(ii) $K \in C\left(L_{\beta}^{2}, L_{\tau}^{2}\right)$ if $\gamma<\beta+1$.

(iii) $K \in C\left(L^{2}\right)$ is selfadjoint.

Proof. (i) (a). Let $\tau \in \boldsymbol{R}$ and $\alpha=\tau /(p-1)$. We get by Hölder,

$$
\begin{aligned}
|(K u)(\xi)|^{p} \leq & \left\{\int_{\boldsymbol{R}^{n}}\left|K\left(\xi, \xi^{\prime}\right)\right|\left(1+\left|\xi^{\prime}\right|\right)^{-\alpha} d \xi^{\prime}\right\}^{p-1} \\
& \times\left\{\int_{\boldsymbol{R}^{n}}\left|K\left(\xi, \xi^{\prime}\right)\right|\left(1+\left|\xi^{\prime}\right|\right)^{\top}\left|u\left(\xi^{\prime}\right)\right|^{p} d \xi^{\prime}\right\} .
\end{aligned}
$$

Choose $\tau>p r$ and apply (4.8) twice with the symmetry of $K\left(\xi, \xi^{\prime}\right)$ taken into account. Then 


$$
\|K u\|_{L_{\gamma}^{p}}^{p} \leq \kappa_{1, \alpha}^{p-1} \kappa_{1, \tau-p(\gamma-1)-1} \int_{\mathbb{R}^{n}}(1+|\xi|)^{p(\gamma-1)}|u(\xi)|^{p} d \xi
$$

which implies (i) (a). The proof of (i) (b) is entirely the same.

(i) (c). Again by (4.8) we get for $\beta \geq 0$,

(4.10) $\quad\|(K u)(\cdot, \xi)\|_{H^{l}\left(\boldsymbol{R}_{x}^{n}\right)} \leq \int_{\boldsymbol{R}^{n}}\left|K\left(\xi, \xi^{\prime}\right)\right|\left\|u\left(\cdot, \xi^{\prime}\right)\right\|_{H^{l}\left(\boldsymbol{R}_{x}^{n}\right)} d \xi^{\prime}$

$$
\leq \kappa_{1, \beta}(1+|\xi|)^{-\beta-1}\|u\|_{l, \beta},
$$

showing $K \in \boldsymbol{B}\left(H_{l, \beta}, H_{l, r}\right)$. To complete the proof, therefore, we have only to repeat the argument of the proof of Lemma 3.2.

(i) (d). The second member in (4.10) can be majorized also by

$$
\left(\int_{\mathbb{R}^{n}}\left|K\left(\xi, \xi^{\prime}\right)\right|^{p^{\prime}}\left(1+\left|\xi^{\prime}\right|\right)^{-p^{\prime} \beta} d \xi^{\prime}\right)^{1 / p^{\prime}}\|u\|_{l, \beta, p} \quad\left(1 / p+1 / p^{\prime}=1\right)
$$

and hence, according to (4.8), it is majorized by

$$
\begin{gathered}
C(1+|\xi|)^{-\left(\beta+1 / p^{\prime}\right)}\|u\|_{l, \beta, p} \quad \text { if } 0<p^{\prime}<n /(n-2) \quad(n \geq 3) \quad \text { or } \\
0<p^{\prime}<2 \quad(n=2) \text {, showing }
\end{gathered}
$$

$$
K \in \mathbb{B}\left(H_{l, \beta, p}, H_{l, \beta}\right) .
$$

Now (i) (d) follows from the fact that $\bigcap_{l, \beta} H_{l, \beta, p}$ is dense in $H_{l, \beta, p}$.

(i) (e) follows from an interpolation theorem applied to (i) (b) with $p$ near 1 and (4.11). Recall here that $H_{l, \beta}=H_{l, \beta, \infty}$.

(ii) Let $\chi_{R}$ be as in (2.11) and denote also by $\chi_{R}$ the multiplication operator induced by the function $\chi_{R}(\xi)$. Let $\gamma<\beta+1$. Then (4.9) implies

$$
\left\|K \chi_{R}\right\|_{B\left(L_{\beta}^{2}, L_{r}^{2}\right)} \leq C(1+R)^{r-\beta-1} \rightarrow 0 \quad(R \rightarrow \infty) .
$$

Also, since $-\beta<-\gamma+1$, we have

$$
\left\|K \chi_{R}\right\|_{B\left(L_{-r}^{2}, L_{-\beta}^{2}\right)} \leq C(1+R)^{r-\beta-1}
$$

as a special case of (4.12). Since $L_{\beta}^{2}$ and $L_{-\beta}^{2}$ are adjoint to each other, and since $K\left(\xi, \xi^{\prime}\right)$ is real symmetric, the adjoint to $K \chi_{R} \in \mathbb{B}\left(L_{-r}^{2}, L_{-\beta}^{2}\right)$ is $\chi_{R} K \in \mathbb{B}\left(L_{\beta}^{2}, L_{\tau}^{2}\right)$ with equal norms. Hence (4.13) implies that

$$
\left\|\chi_{R} K\right\|_{B\left(L_{\beta}^{2}, L_{\tau}^{2}\right)} \rightarrow 0 \quad(R \rightarrow \infty),
$$

which, together with (4.12), shows, putting $K_{R}=\left(I-\chi_{R}\right) K\left(I-\chi_{R}\right)$.

$$
\left\|K-K_{R}\right\|_{B\left(L_{\beta}^{2}, L_{r}^{2}\right)} \rightarrow 0 \quad(R \rightarrow \infty),
$$


Thus (ii) can be concluded if $K_{R} \in C\left(L_{\beta}^{2}, L_{\tau}^{2}\right)$, [8. Theorem III. 4. 7]. Let $K_{R, \varepsilon}$ be the integral operator induced by the kernel $\chi_{\varepsilon}\left(\xi-\xi^{\prime}\right)\left\{1-x_{R}(\xi)\right\}$ $\times K\left(\xi, \xi^{\prime}\right)\left\{1-x_{R}\left(\xi^{\prime}\right)\right\}$. Then $\Lambda^{r} K_{R, \varepsilon} \Lambda^{-\beta}$ is of Hilbert-Schmidt type, that

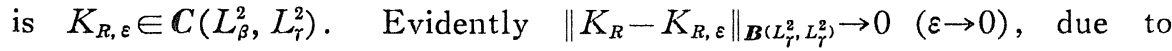
(4.3), (4.5) and (4.6). This implies that $K_{R} \in C\left(L_{\beta}^{2}, L_{r}^{2}\right)$. Finally (iii) is obvious and this completes the proof of Lemma 4.2.

Finally we should state some properties of the operator $L$ of (3.3) considered in the space $L^{2}=L^{2}\left(\boldsymbol{R}_{\xi}^{n}\right)$. We note from (4.1) and (4.2) that

$$
L=-\Lambda+K \text {. }
$$

Then $L \in \boldsymbol{B}\left(L^{2}\right)$ in virtue of Lemmas 4.1 (i) and 4.2 (i) (a).

Lemma 4. 3. Let $L$ be as above.

(i) $L$ is nonnegative selfadjoint on $L^{2}$.

(ii) $\sigma_{e}(L)=\sigma_{e}(\Lambda) \subset\left[-\nu_{0}, 0\right], \sigma_{d}(L) \subset(-\infty, 0] \backslash \sigma_{e}(L)$ where $\nu_{0}=\sup \nu(\xi)$.

(iii) $0 \in \sigma_{p}(L)$ with multiplicity $n+2$ whose eigenspace is spanned by the functions

$$
\begin{aligned}
& \varphi_{0}(\xi)=g(\xi)^{1 / 2}, \varphi_{j}(\xi)=\xi_{j} g(\xi)^{1 / 2}, 1 \leq j \leq n, \\
& \varphi_{n+1}(\xi)=|\xi|^{2} g(\xi)^{1 / 2} .
\end{aligned}
$$

(iv) Denote by $P$ the eigenprojection for $0 \in \sigma_{p}(L)$. Then $P \in \boldsymbol{C}\left(L_{\alpha}^{2}, L_{\gamma}^{2}\right)$ for any $\beta, r \in \boldsymbol{R}$.

Proof. The selfadjointness of $L$ and the assertion (ii) follow from Lemmas 4.1 (ii) and 4.2 (iii), [7. Theorem IV.5.35]. The proof of the nonnegativity and that of (iii) will be referred to [3], and (iv) is obvious from the fact that $\varphi_{j}$ of $(4.16)$ are rapidly decreasing when $|\xi| \rightarrow \infty$.

Let $r \in O(n)$ be a rotation in $\boldsymbol{R}_{\xi}^{n}$ and define $R$ by

$$
(R u)(\xi)=u(r \xi)
$$


Lemma 4. 4. $\Lambda, K$ and $L$ commute rith $R$.

This is a conscquence of the fact that $q$ in (1.2) is a function only of $\left|\xi-\xi^{\prime}\right|$ and $\theta$. For the proof, see [3].

A formal calculation gives the

Lemma 4. 5. Let $u$ and $v$ be in a suitable space such that $\Gamma[u$, $v] \in L_{a}^{2}$. Then $P \Gamma[u, v]=0$, where $P$ is as in Lemma 4.3 (iv).

\section{§ 5. Operator's $\mathcal{H}(\boldsymbol{k})$ and $B_{0}(\boldsymbol{k})$}

To derive decay estimate for $e^{t B}$, we shall appeal to the Fourier transformation. Recall that $L$ acts only on $\xi$. Then (3.7) and (3.9) yield

$$
(B u)^{\wedge}(k, \xi)=-i\langle k, \xi\rangle \widehat{u}(k, \xi)+L \widehat{u}(k, \xi) .
$$

The right side is a multiplication operator with respect to $k$. Therefore, regarding $k$ as a parameter, we define

$$
(B(k) u)(\xi)=-i\langle k, \xi\rangle u(\xi)+L u(\xi)
$$

for each $k \in \boldsymbol{R}^{n}$. For our purpose this operator should be investigated in $L_{\beta}^{2}$ for various values of $\beta \in \mathbb{R}$. We will do this by perturbation technics, introducing auxiliary operators

$$
\begin{aligned}
& A_{0}(k)=-i\langle k, \xi\rangle \times, \\
& A(k)=A_{0}(k)+A \\
& B_{0}(k)=A_{0}(k)+L-P=A(k)+K-P=B(k)-P,
\end{aligned}
$$

where $A, K$ and $P$ are as in (4.1), (4.2) and Lemma 4.3 (iv) respectively. They should also be studied in $L_{\beta}^{2}$ for all $\beta \in \mathbb{R}$. This will be done in this section, while $B(k)$ will be discussed in the following two sections.

Endowed with the domain

$$
D\left(A_{0}(k)\right)=\left\{u \in L_{\beta}^{2} ;\langle k, \xi\rangle u(\xi) \in L_{\beta}^{2}\right\},
$$

$A_{0}(k)$ is anti-selfadjoint in the Hilbert space $L_{\beta}^{2}$ for any $\beta \in \mathbb{R}$. Hence it generates a unitary group $e^{i A_{0}(k)}$ for each $k$. Further, if $k \neq 0$, 


$$
\sigma\left(A_{0}(k)\right)=\sigma_{e}\left(A_{0}(k)\right)=i \boldsymbol{R}, \quad \sigma_{p}\left(A_{0}(k)\right)=\phi,
$$

where $i \boldsymbol{R}=\{\lambda \in C ; \operatorname{Re} \lambda=0\}$. Note that $A_{0}(0)=0$. Define

$$
D(A(k))=D\left(B_{0}(k)\right)=D\left(A_{0}(k)\right) .
$$

Since $\Lambda, L, P \in \boldsymbol{B}\left(L_{\beta}^{2}\right)$ by Lemmas 4.1 (i), 4.2 (i) and 4.3 (iv), this choice of domains are maximal in $L_{\beta}^{2}$, and moreover since $A_{0}(k)$ is a generator, $A(k)$ and $B_{0}(k)$ are also generators [7. Theorem IX. 2.1]. Note also that

$$
A(k)^{*}=A(-k), \quad B_{0}(k)^{*}=B_{0}(-k) .
$$

The semigroup $e^{t A(k)}$ is a contraction semigroup given as

$$
e^{t A(k)}=e^{-t(i\langle k, \xi\rangle)+\nu(\xi))} \times
$$

and the resolvent is

$$
(\lambda-A(k))^{-1}=(\lambda+\nu(\xi)+i\langle k, \xi\rangle)^{-1} \times .
$$

Since $\nu(\xi) \geq 0, \nu(\xi) \rightarrow 0(|\xi| \rightarrow \infty)$ by (4.4), we see easily

$$
\begin{gathered}
i \boldsymbol{R} \subset \sigma(A(k))=\sigma_{e}(A(k))=\overline{\left\{i\langle k, \xi\rangle+\nu(\xi) ; \xi \in \boldsymbol{R}^{n}\right\}} \subset \overline{\boldsymbol{C}}_{-}, \\
\rho(A(k)) \supset \boldsymbol{C}_{+},
\end{gathered}
$$

where $\boldsymbol{C}_{=}=\{\lambda \in \boldsymbol{C} ; \operatorname{Re} \lambda \gtrless 0\}$ (same sign). In particular $i \boldsymbol{R}$ is a part of the boundary of $\sigma(A(k))$, if $k \neq 0$.

The resolvent $(5.11)$ is in $\boldsymbol{B}\left(L_{\beta}^{2}\right)$ for $\lambda \in \rho(A(k))$ by definition and hence in particular for $\lambda \in C_{\perp}$ by $(5.12)$. Now we note that (5.11) can be considered also as an operator in $\boldsymbol{B}\left(L_{\alpha}^{2}, L_{\beta}^{2}\right)$ as far as $\alpha \geq \beta$. This point of view is advantageous since even $\lambda \in i \boldsymbol{R}$ is then acceptable in (5.11), which is of course impossible as an operator of $\boldsymbol{B}\left(L_{\beta}^{2}\right)$ by $(5.12)$. More precisely, we now prove the

\section{Lemma 5.1. Put}

$$
\Sigma \equiv \Sigma_{(\lambda, k)}=\boldsymbol{C}_{+} \times \boldsymbol{R}^{n}
$$

and write $R(\lambda, k)=(\lambda-A(k))^{-1}$. Let $\delta$ be as in [Q] (ii).
(i) $R(\lambda, k) \in L^{\infty}\left(\bar{\Sigma} ; \boldsymbol{B}\left(L_{\alpha}^{2}, L_{\beta}^{2}\right)\right)$ if $\alpha \geq \beta+\delta$.
(ii) $R(\lambda, k) \in \mathscr{B}^{0}\left(\bar{\Sigma} ; \boldsymbol{B}\left(L_{\alpha}^{2}, L_{\beta}^{2}\right)\right)$ if $\alpha>\beta+\delta$.
(iii) For each fixed $r>0$ and $u \in L_{\beta+\delta}^{2}$, 


$$
\sup _{\lambda \in \bar{C}_{+},|\lambda| \geq a,|k| \leq r}\|R(\lambda, k) u\|_{L_{\beta}^{2} \rightarrow 0} \quad(a \rightarrow \infty) .
$$

(iv) Write $\lambda=\gamma+i \tau$ with $\gamma, \tau \in \mathbb{R}$, and let $u \in L_{\beta+\delta / 2}^{2}$. Then

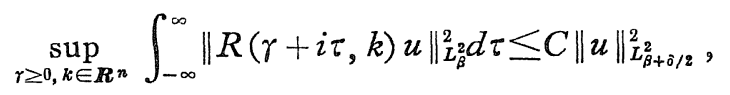

with some constant $C \geq 0$ not depending on $u$.

Proof. (i) If we define

$$
f(\lambda, k, \xi)=(1+|\xi|)^{-\delta}(\lambda+\nu(\xi)+i\langle k, \xi\rangle)^{-1},
$$

then we see, on one hand

$$
\|R(\lambda, k)\|_{\boldsymbol{B}\left(L_{\alpha}^{2}, L_{\beta}^{2}\right)}=\sup _{\xi}(1+|\xi|)^{\beta+\delta-\alpha}|f(\lambda, k, \xi)|
$$

by (5.11), and on the other hand

$$
|f| \leq \nu_{-1} \nu(\xi) /(\operatorname{Re} \lambda+\nu(\xi)) \leq \nu_{-1}
$$

for all $(\lambda, k) \in \bar{\Sigma}$ and $\xi \in \boldsymbol{R}^{n}$ by (4.3), proving (i).

(ii) Let $\chi_{m}=\chi_{m}(\xi)$ be the same as in (2.11). By (5.14) and (5.15), we see that

$$
\left\|R(\lambda, k) \chi_{m}\right\|_{\boldsymbol{B}\left(L_{\alpha^{\prime}}^{2}, L_{\beta}^{2}\right)} \leq \nu_{-1}(1+m)^{\beta+\delta-\alpha} \rightarrow 0 \quad(m \rightarrow \infty)
$$

uniformly in $(\lambda, k) \in \bar{\Sigma}$ if $\alpha>\beta+\delta$. On the other hand

$$
\begin{aligned}
\mid f(\lambda, k, \xi)- & f\left(\lambda^{\prime}, k^{\prime}, \xi\right) i \leq|f(\lambda, k, \xi)|\left|f\left(\lambda^{\prime}, k^{\prime}, \xi\right)\right| \\
& \times(1+|\xi|)^{\delta}\left(\left|\lambda-\lambda^{\prime}\right|+|\xi|\left|k-k^{\prime}\right|\right)
\end{aligned}
$$

which, together with (5.14) and (5.15) again, shows that $R(\lambda, k)\left(I-\chi_{m}\right)$ $\in \mathcal{B}^{\circ}\left(\bar{\Sigma} ; \boldsymbol{B}\left(L_{\beta}^{2}\right)\right)$ for each fixed $m$. Now (ii) is evident from this and (5. 16).

(iii) Let $|k| \leq r,|\xi| \leq m$ and $|\operatorname{Im} \lambda| \geq r m$. Then $|\operatorname{Im} \lambda-\langle k, \xi\rangle| \geq|\operatorname{Im} \lambda|$ $-r m$, and thereby we find that

$$
\begin{gathered}
\sup _{|k| \leq r}\left\|R(\lambda, k)\left(I-\chi_{m}\right)\right\|_{B\left(L_{\beta}^{2}\right)} \leq C(\operatorname{Re} \lambda+|\operatorname{Im} \lambda|-r m)^{-1} \rightarrow 0 \\
\left(|\lambda| \rightarrow \infty, \lambda \in \overline{\mathbb{C}}_{+}\right)
\end{gathered}
$$

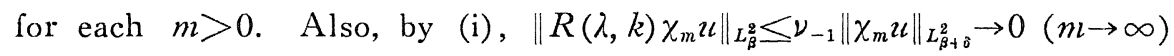
uniformly in $(\lambda, k) \in \bar{\Sigma}$ for each $u \in L_{\beta+\delta}^{2}$. Combining these results, we 
have (iii).

(iv) is a simple consequence of

$$
\begin{aligned}
\int_{-\infty}^{\infty}|f(\gamma+i \tau, k, \xi)|^{2} d \tau & =(1+|\xi|)^{-2 \delta} \int_{-\infty}^{\infty}\left\{(\gamma+\nu(\xi))^{2}+\tau^{2}\right\}^{-1} d \tau \\
& =\pi(1+|\xi|)^{-2 \delta}|\gamma+\nu(\xi)|^{-1} \leq \pi \nu \nu_{-1}(1+|\xi|)^{-\delta} .
\end{aligned}
$$

Remark 5.1. Since $L_{\alpha}^{2}$ is dense in $L_{\beta}^{2}$ if $\alpha \geq \beta$, it follows from Lemmas 5.1 (i) and (ii) that $R(\lambda, k) u \in \mathscr{B}^{0}\left(\Sigma ; L_{\beta}^{2}\right)$ whenever $u \in L_{\beta+\delta}^{2}$.

Now we study $B_{0}(k)$.

Lemma 5.2. $B_{0}(k)$ is dissipative in $L^{2}=L_{0}^{2}$.

Proof. In virtue of Lemma $4.3, L-P$ is bounded, selfadjoint and negative on $L^{2}$. This and the anti-selfadjointness of $A_{0}(k)$ implies that for any $u \in D\left(B_{0}(k)\right)=D\left(A_{0}(k)\right), u \neq 0$,

$$
\operatorname{Re}\left(B_{0}(k) u, u\right)_{L^{2}}=((L-P) u, u)<0,
$$

which proves the lemma.

We noted already that $B_{0}(k)$ is a generator. This lemma, therefore, shows that $B_{0}(k)$ is maximal dissipative in $L^{2}$, or equivalently, $e^{t B_{0}(k)}$ is a contraction semigroup on $L^{2} . \quad B_{0}(k)$ is not dissipative in $L_{\beta}^{2}$ if $\beta \neq 0$, but $e^{t B_{0}(k)}$ can be shown to be a bounded semigroup. This fact, however, is not used in the sequel, and so the proof will not be given. Instead, we need some properties of $\sigma\left(B_{0}(k)\right)$ in $L_{\beta}^{2}$ for arbitrary $\beta$, namely,

Proposition 5. 1. (i) $\sigma\left(B_{0}(k)\right) \subset \overline{\boldsymbol{C}}_{-}, \rho\left(B_{0}(k)\right) \supset \boldsymbol{C}_{+}$. (ii) $\sigma_{e}\left(B_{0}(k)\right)$ $=\sigma_{e}(A(k)) . \quad$ (iii) $\sigma_{p}\left(B_{0}(k)\right) \subset C_{-}$.

Proof. If we put $K_{0}=K-P$, then

$$
B_{0}(k)=A(k)+K_{0}
$$

by (5.5), while by Lemmas 4.2 (i) (a), (ii) and 4.3 (iv), we see for any $\varepsilon>0$,

$$
K_{0} \in \boldsymbol{B}\left(L_{\beta}^{2}, L_{\beta+1}^{2}\right) \cap \boldsymbol{C}\left(L_{\beta}^{2}, L_{\beta+1-\varepsilon}^{2}\right) \subset \boldsymbol{C}\left(L_{\beta}^{2}\right)
$$


Therefore (ii) is a simple application of [7. Theorem IV. 5.35]. Moreover (5.19) implies $\sigma\left(B_{0}(k)\right) \cap \rho(A(k)) \subset \sigma_{d}\left(B_{0}(k)\right)$. Consequently (i) follows from (iii) in virtue of (5.12) and (ii). To prove (iii), let $\lambda$ $\in \sigma_{p}\left(B_{0}(k)\right)$. Then $\lambda u=B_{0}(k) u$ with some $u \in D\left(B_{0}(k)\right), u \neq 0$. If $\beta \geq 0$, then $u \in L^{2}=L_{0}^{2}$, and (5.17) can be applied to conclude $\operatorname{Re} \lambda<0$. Let $\beta<0$ and suppose $\operatorname{Re} \lambda>0$. Then $K_{0} u \in L_{\beta+1}^{2}$ by (5.19), so that $u=(\lambda$ $-A(k))^{-1} K_{0} u \in L_{\beta+1-\delta}^{2}$ in view of Lemma 5.1 (i). Since $\delta<1$, this argument can be repeated to verify $u \in \bigcap_{\beta} L_{\beta}^{2}$. This shows that $u \in D\left(B_{0}(k)\right)$ in $L^{2}$. Hence $\operatorname{Re} \lambda<0$ as proved earlier. This completes the proof of the proposition.

Let $\lambda \in C_{+}$. Then $\lambda \in \rho\left(B_{0}(k)\right) \cap \rho(A(k))$ by (5.12) and Proposition 5.1 (i), and we can easily obtain from (5.18)

$$
\left(\lambda-B_{0}(k)\right)^{-1}=\left(I-(\lambda-A(k))^{-1} K_{0}\right)^{-1}(\lambda-A(k))^{-1} .
$$

Write

$$
F(\lambda, k)=(\lambda-A(k))^{-1} K_{0}
$$

Lemma 5.3. Let $\Sigma=C_{+} \times \mathbb{R}^{n}$, and $\beta \in \mathbb{R}^{n}$.

(i) $F(\lambda, k) \in \mathscr{B}^{0}\left(\bar{\Sigma} ; \mathbb{C}\left(L_{\beta}^{2}\right)\right)$.

(ii) $\sup _{\lambda \in \overline{\boldsymbol{C}}_{+},|\lambda| \geq b,|k| \leq r}\|F(\lambda, k)\|_{\boldsymbol{B}\left(L_{\beta}^{2}\right) \rightarrow 0 \quad(b \rightarrow \infty)}$

for each $r>0$.

(iii) $\sup _{\lambda \in \overline{\boldsymbol{C}}_{+},|k| \geq r}\|F(\lambda, k)\|_{\boldsymbol{B}\left(L_{\beta}^{2}\right) \rightarrow 0 \quad(r \rightarrow \infty) .}$

Proof. Recall $\delta<1$. Then (i) follows from Lemma 5.1 (ii), (5.19) and [7. Theorem III. 4. 8], while (ii) from Lemma 5.1 (iii), (5. 19) and [7. Lemma III. 3.7]. To prove (iii), let $\chi_{m}=\chi_{m}(\xi)$ be as in (2.11). In view of $(5.16)$ and $(5.19)$, we have

$$
\left\|(\lambda-A(k))^{-1} \chi_{m} K_{0}\right\|_{\boldsymbol{B}\left(L_{\beta}^{2}\right)} \leq C(1+m)^{-(1-\delta)},
$$

the right side of which tend to zero as $m \rightarrow \infty$ uniformly in $(\lambda, k) \in \bar{\Sigma}$. Hence if we put $I_{m}(\lambda, k)=(\lambda-A(k))^{-1}\left(I-X_{m}\right)$, then it suffices to prove that $\left\|I_{m}(\lambda, k) K_{0}\right\|_{\boldsymbol{B}\left(L_{\beta}^{2}\right) \rightarrow 0}(|k| \rightarrow \infty)$ uniformly in $\lambda \in \overline{\mathbb{C}}_{+}$for each $m>0$, 
and this follows, owing to Lemma 5.1 (i) and (5.19), if

$$
\sup _{\lambda \in \overline{\boldsymbol{C}_{+},|k| \geq r}}\left\|I_{m}(\lambda, k) u\right\|_{L_{\beta}^{2} \rightarrow 0} \quad(r \rightarrow \infty)
$$

for each $u \in L_{\beta+\delta}^{2}$ and $m>0$, [7. Lemma III. 3.7]. To prove this, put

$$
\begin{aligned}
& S^{1}=\left\{\xi \in \boldsymbol{R}^{n} ;|\xi| \leq m,|\operatorname{Im} \lambda-\langle k, \xi\rangle| \leq|k| / \sqrt{r}\right\}, \\
& S^{2}=\left\{\xi \in \boldsymbol{R}^{n} ;|\xi| \leq m\right\} \backslash S^{1}
\end{aligned}
$$

for each $(\lambda, k) \in \bar{\Sigma}$ and $r, m>0$. It is not difficult to see that mes $S^{1}$ $\leq C m^{n-1} / \sqrt{r}$ so that

$$
\operatorname{mes} S^{1} \rightarrow 0 \quad(r \rightarrow \infty)
$$

uniformly in $(\lambda, k) \in \bar{\Sigma}$ for each fixed $m>0$. Moreover if $f$ is the same as in the proof of Lemma 5.1, then $|f| \leq \nu_{-1}$ in $S^{1}$ and $|f| \leq 1 /(|k| / \sqrt{r})$ $\leq 1 / \sqrt{r}$ in $S^{2}$ if $|k| \geq r$. Hence

$$
\begin{aligned}
\left\|I_{m}(\lambda, k) u\right\|_{L_{\beta}^{2}}^{2}= & {\left[\int_{S^{1}}+\int_{S^{2}}\right]|f(\lambda, k, \xi)|^{2}(1+|\xi|)^{2(\beta+\delta)}|u(\xi)|^{2} d \xi } \\
& \leq \nu_{-1}\|u\|_{L_{\beta+\delta}^{2}\left(S^{1}\right)}^{2}+\frac{1}{r}\|u\|_{L_{\beta+\delta}^{2}}
\end{aligned}
$$

for $\lambda \in \overline{\boldsymbol{C}}_{+}$and $|k| \geq r$. Now (5.22) follows from this and (5.23).

Lemma 5. 4. (i) $1 \in \rho(F(\lambda, k))$ for all $(\lambda, k) \in \bar{\Sigma}$. (ii) (I$F(\lambda, k))^{-1} \in \mathscr{B}^{0}\left(\bar{\Sigma} ; \boldsymbol{B}\left(L_{\beta}^{2}\right)\right)$.

Proof, (i) Suppose $1 \in \sigma(F(\lambda, k))$ for some $(\lambda, k) \in \bar{\Sigma}$, Then $1 \in \sigma_{d}(F(\lambda, k))$ by Lemma 5.3 (i). So $u=F(\lambda, k) u$ with some $u \in L_{\beta}^{2}$, $u \neq 0$. Then $u \in L_{\beta+1-\delta}^{2}$ and therefore $u \in \bigcap_{\beta} L_{\beta}^{2}$ and $\lambda \in \sigma_{p}\left(B_{0}(k)\right)$ as shown in the proof of Proposition 5.1, a contradiction to Proposition 5.1 (iii), proving (i).

(ii) (i) and Lemma 5.3 (i) imply $(I-F(\lambda, k))^{-1} \in C^{0}\left(\bar{\Sigma} ; \boldsymbol{B}\left(L_{\beta}^{2}\right)\right)$. so that it suffices to show that $(I-F(\lambda, k))^{-1} \in L^{\infty}\left(\bar{\Sigma}_{r} ; \boldsymbol{B}\left(L_{\beta}^{2}\right)\right)$ for some $r>0$ where we have put

$$
\Sigma_{r}=\{\lambda \in \Sigma ;|\lambda|+|k| \geq r\} .
$$

And this follows from Lemma 5.3 (ii) and (iii). In fact there is an $r>0$ such that $\|F(\lambda, k)\|_{\boldsymbol{B}\left(L^{2}\right)} \leq 1 / 2$ for all $(\lambda, k) \in \bar{\Sigma}_{r}$. Hence $\|(I-$ 
$F(\lambda, k))^{-1} \|_{\boldsymbol{B}\left(L_{\beta}^{2}\right)} \leq 2$ by Neuman series.

Applying Lemmas 5.1 and 5.4 to $(5.20)$, we readily have

Lemma 5.5. Lemma 5.1 is valid with $B_{0}(k)$ in place of $A(k)$.

The assertions (i), $\cdots$, (iv) of Lemma 5.1 for $R(\lambda, k)=\left(\lambda-B_{0}(k)\right)^{-1}$ will be referred to as Lemma 5.5 (i), ..., (iv) respectively.

\section{$\S 6$. Estimates of $e^{t B(k)}$ for $|\boldsymbol{k}|$ Bounded Away from Zero}

Choose $D(A(k))$ of $(5.8)$ as the domain $D(B(k))$ and write

$$
B(k)=B_{0}(k)+P=A_{0}(k)+L .
$$

Since $B_{0}(k)$ is a semigroup generator, so is $B(k)$ in $L_{\beta}^{2}$ by Lemma 4.3 (iv). Moreover we have the

Lemma 6. 1. $e^{t B(k)}$ is a contraction semigroup on $L^{2}=L_{0}^{2}$ for each $k \in \boldsymbol{R}^{n} ;\left\|e^{t B(k)}\right\|_{\boldsymbol{B}\left(L^{2}\right)} \leq 1$.

Proof. Instead of (5.17) we have by Lemma 4.3 (i),

$$
\operatorname{Re}(B(k) u, u)_{L^{2}}=(L u, u)_{L^{2}} \leq 0, u \in D(B(k)),
$$

showing that $B(k)$ is dossipative in $L^{2}$, whence the lemma follows according to the remark given below Lemma 5.2.

The following proposition, corresponding to Proposition 5.1, holds in $L_{\beta}^{2}$ for all $\beta \in \boldsymbol{R}$.

Proposition 6.1. (i) $\sigma(B(k)) \subset \overline{\mathbb{C}}_{-}, \rho(B(k)) \supset \mathbb{C}_{+}$(ii) $\sigma(B(k))$ $=\sigma\left(B_{0}(k)\right) . \quad$ (iii) $\sigma_{p}(B(k)) \subset \mathbb{C}_{-}$for $k \neq 0$.

Proof. The only difference from Proposition 5.1 is the restriction $k \neq 0$ in (iii). Indeed $0 \in \sigma_{\rho}(B(0))=\sigma_{p}(L)$ by Lemma 4.3 (iii). Let $k \neq 0$, and $\lambda \in \sigma_{p}(B(k))$. Then $\lambda u=B(k) u$ with some $u \in D(B(k)), u \neq 0$. Consequently $\operatorname{Re} \lambda \leq 0$ by (6.2). If $\operatorname{Re} \lambda=0$, then by (6.2) again and by Lemma 4.3 (i), $L u=0$, and so $\lambda u=A_{0}(k) u$, that is, $\lambda \in \sigma_{p}\left(A_{0}(k)\right)$, 
a contradiction to (5.7). Taking account of this fact, we can repeat the proof of Proposition 5.1 to conclude the proposition.

Let $\lambda \in \boldsymbol{C}_{+}$. Then $\lambda \in \rho(B(k)) \cap \rho\left(B_{0}(k)\right)$ and by (6.1)

$$
(\lambda-B(k))^{-1}=\left(I-\left(\lambda-B_{0}(k)\right)^{-1} P\right)^{-1}\left(\lambda-B_{0}(k)\right)^{-1} .
$$

Put

$$
G(\lambda, k)=\left(\lambda-B_{0}(k)\right)^{-1} P=(I-F(\lambda, k))^{-1}(\lambda-A(k))^{-1} P .
$$

In view of Lemma 4.3 (iv), Lemma 5.3 applies also to $(\lambda-A(k))^{-1} P$. Hence by Lemma 5.4 and (6.4), this is the case also for $G(\lambda, k)$.

Lemma 6. 2. Lemma 5.3 is valid if $F(\lambda, k)$ is replaced by $G(\lambda, k)$.

Now the following two lemmas follow from Proposition 6.1 and Lemmas 5.5 and 6.2 applied to (6.3), just in the same way as Lemmas 5.4 and 5.5 followed from Proposition 5.1 and Lemmas 5.1 and 5.3 applied to $(5,20)$.

I.emma 6. 3. For any $r>0$, (i) $1 \in \rho(G(\lambda, k))$ for all $(\lambda, k) \in \bar{\Sigma}_{r}$, and (ii) $(1-G(\lambda, k))^{-1} \in \mathscr{B}^{0}\left(\bar{\Sigma}_{r} ; \boldsymbol{B}\left(L_{\beta}^{2}\right)\right)$, where $\Sigma_{r}$ was defined by (5.25).

Lemma 6. 4. Put $R(\lambda, k)=(\lambda-B(k))^{-1}$, and let $r>0$. Then Lemma 5.1 is valid if $\Sigma$ is replaced by $\Sigma_{r}$ in (i) and (ii), and if the supremum in (iv) is taken for $r \geq 0$ and $|k| \geq r$. The constant $C$ in (iv) depends, of course, on $r$.

The assertions (i) to (iv) of Lemma 5.1 for $R(\lambda, k)=(\lambda-B(k))^{-1}$ will be referred to as Lemma 6.4 (i) to (iv) respectively. In the above, $r=0$ is not possible because of the restriction $k \neq 0$ in Proposition 6.1 (iii).

Now Lemma 6.4 enable us to evaluate $e^{t B(k)}$ for $k \neq 0$.

Theorem 6.1. Let $\beta \geq 0$ and $r>0$. There exists a constant 
$C \geq 0$ such that for any $l \geq 0$ and $k \in \mathbb{R}^{n},|k| \geq r$

$$
\left\|e^{t B(k)}\right\|_{B\left(L_{\beta}^{2}, L^{2}\right)} \leq C(1+t)^{-\beta / \delta}
$$

where $\delta>0$ is as in [Q] (ii), and $L^{2}=L_{0}^{2}$.

Proof. If $\beta=0$, this is nothing but Lemma 6.]. For $\beta>0$, it is sufficient to deal only with the case $\beta=l \delta, l \in \mathbb{N}$. For, the theorem for general $\beta \geq 0$ then follows from an interpolation theorem.

For simplification of notations we write $R(\lambda)=(\lambda-B(k))^{-1}$ and denote the inner product and the norm of $L_{\beta}^{2}$ as $(\cdot, \cdot)_{\beta}$ and $\|\cdot\|_{\beta}$ respectively.

To prove the theorem, we use the fact that $e^{t B(k)}$ is given as the inverse Laplace transformation of the resolvent $R(\lambda)=(\lambda-B(k))^{-1}$;

$$
e^{t B(k)} u=s-\lim _{a \rightarrow \infty} \frac{1}{2 \pi i} \int_{\tau-i a}^{\gamma+i a} e^{\lambda t} R(\lambda) u d \lambda
$$

Here $u \in D(B(k))$ and $\iota>0$, and $\gamma>0$ can be chosen arbitrarily. We first show that we can put $\gamma=0$.

Let $\beta \geq 0$ and $l \in N$, and let $u \in L_{\beta+l \delta}^{2}$. By an argument similar to that given in Remark 5.1, we see from Lemma 6.4 (i) (ii) (iii) that for any $r>0$

$$
R(\lambda)^{l} u \in \mathscr{B}^{0}\left(\bar{\Sigma}_{r} ; L_{\beta}^{2}\right),
$$

and that for any $k \in \boldsymbol{R}$, including $k=0$,

$$
\sup _{\lambda \in \overline{\boldsymbol{C}}_{+},|\lambda| \geq b}\left\|R(\lambda)^{l} u\right\|_{\beta} \rightarrow 0 \quad(b \rightarrow \infty) .
$$

Since $R(\lambda)$ is a resolvent, $R(\lambda) u$ is analytic in $\lambda$ in $\rho(B(k))$ and hence in particular in $C_{+}$by Proposition 6.1 (i). Thus Cauchy's theorem on the integrals of holomorphic functions can be applied to the right side of (6.5) to shift the path of integration to the imaginary axis by the aid of (6.6) and (6.7) with $l=1$. Thus $r=0$ is possible in (6.5) if $u \in L_{\beta+\delta}^{2}$.

Therefore, defining

$$
I_{l}(t, a, b)=\int_{a}^{b} e^{i \tau t} R(i \tau)^{l+1} d \tau, \quad l \in \mathbb{N}
$$

we have 


$$
e^{t B(k)} u=\frac{1}{2 \pi} s-\lim _{a \rightarrow \infty} I_{0}(t,-a, a) u
$$

for $t>0$ and $u \in L_{\beta+\delta}^{2} \cap D(B(k))$.

It is well-known that $d^{l} R(\lambda) / d \lambda^{l}=(-1)^{l} l ! R(\lambda)^{l+1}$ for $\lambda \in \rho(B(k))$, $l \in \boldsymbol{N}$, and so we have, putting $\lambda=\gamma+i \tau$,

$$
\frac{d^{l}}{d \gamma^{l}} R(\gamma+i \tau) u=\frac{1}{i^{l}} \frac{d^{l}}{d \tau^{l}} R(\gamma+i \tau) u=(-1)^{l} l ! R(\gamma+i \tau)^{l+1} u
$$

This holds for $u \in L_{\beta}^{2}$ and $\gamma+i \tau \in \rho(B(k)) \supset C_{+}$, but if $u \in L_{\beta+(l+1) \delta}^{2}$, this is valid even for $\gamma=0$ in virtue of (6.6). Therefore we can integrate $I_{0}(t, a, b)$ by parts to obtain

$$
I_{0}(t, a, b) u=\left[\sum_{k=1}^{l-1} \frac{e^{i \tau t}}{t^{k}} k ! R(i \tau)^{k+1} u\right]_{\tau=a}^{\tau=b}+\frac{l !}{t^{l}} I_{l}(t, a, b) u
$$

where $t>0$ and $u \in L_{\beta+(l+1) \delta}^{2}$. The first term of the right side tends to zero as $a \rightarrow-\infty, b \rightarrow \infty$ by $(6.7)$. Consequently (6.9) reduces to

$$
e^{t B(k)} u=\frac{l !}{2 \pi t^{l}} s-\lim _{a \rightarrow \infty} I_{l}(t,-a, a) u
$$

if $t>0$ and $u \in \mathrm{L}_{\beta+(l+1) \delta}^{2} \cap D(B(k))$.

To evaluate the last limit, observe that

$$
\begin{aligned}
\left|\left(R(\lambda)^{l+1} u, v\right)_{\beta}\right| & =\left|\left(R(\lambda)^{l} u, R(\lambda)^{*} v\right)_{\beta}\right| \\
& \leq\left\|R(\lambda)^{\imath} u\right\|_{\beta+\delta / 2}\left\|R(\lambda)^{*} v\right\|_{\beta-\delta / 2} \\
& \leq C\|R(\lambda) u\|_{\beta+(l-1 / 2) \delta}\left\|R(\lambda)^{*} v\right\|_{\beta-\delta / 2}
\end{aligned}
$$

holds for $l \geq 1$ and $(\lambda, k) \in \bar{\Sigma}_{r}$ where

$$
C \equiv \sup _{(\lambda, k) \in \bar{\Sigma}_{r}}\left\|R(\lambda)^{l-1}\right\|_{B\left(L_{\beta+(l-1 / 2) \delta}^{2}, L_{\beta+\delta / 2}^{2}\right)}<+\infty
$$

by Lemma 6.4 (i). Since $R(\lambda)^{*}=(\bar{\lambda}-B(-k))^{-1}$ in view of $(5.9)$, it follows from Lemma 6.4 (iv) and (6.13) that

$$
\begin{aligned}
\left|(I(t, a ; b) u, v)_{\beta}\right| \leq \int_{a}^{b}\left|\left(R(i \tau)^{l+1} u, v\right)_{\beta}\right| d \tau \\
\quad \leq C\left(\int_{a}^{b}\|R(i \tau) u\|_{\beta+(l-1 / 2) \delta}^{2} d \tau\right)^{1 / 2}\left(\int_{a}^{b}\left\|R(i \tau)^{*} v\right\|_{\beta-\delta / 2}^{2} d \tau\right)^{1 / 2} \\
\quad \leq C_{0}\|u\|_{\beta+l \delta}\|v\|_{\beta},
\end{aligned}
$$


for all $t>0$ and $|k| \geq r$. Note that if $|k| \geq r$, then $(\lambda, k) \in \Sigma_{r}$ for all $\lambda \in \overline{\boldsymbol{C}}_{+}$. In (6.14), the constant $C_{0}$ depends only on $r$. Now (6.14) implies not only that $I_{l}(t, a, b)$ converges as $a \rightarrow-\infty, b \rightarrow \infty$ absolutely in the weak topology of $\mathbb{B}\left(L_{\beta+1 \delta}^{2}, L_{\beta}^{2}\right)$ to some $I^{(l)}(t) \in \mathbb{B}\left(L_{\beta+l \delta}^{2}, L_{\beta}^{2}\right)$ for all $t \geq 0$, but also the limit $I^{(l)}(t)$ is uniformly bounded there in $t \geq 0$ and $|k| \geq r$. Noting that $L_{\beta+(l+1) \delta}^{2}$ is dense in $L_{\beta+l \delta}^{2}$, we therefore obtain by $(6.12)$,

$$
\sup _{|k| \geq r}\left\|e^{t B_{0}(k)}\right\|_{\mathbb{B}\left(L_{\beta+l l}^{2}, L_{\beta}^{2}\right)} \leq C t^{-l} .
$$

Put $\beta=0$ here and use Lemma 6.1 to complete the proof of the theorem.

Remark 6.1. In $L_{\beta}^{2}, \beta \neq 0, e^{t B(k)}$ is not a contraction semigroup but it can be shown to be bounded in $\mathbb{B}\left(L_{\beta}^{2}\right)$ uniformly in $t \geq 0$ and $k \in \mathbb{R}^{n}$. Hence Theorem 6.1 is valid even with $\mathbb{B}\left(L_{\alpha}^{2}, L^{2}\right)$ replaced by $\mathbb{B}\left(L_{\beta+\alpha}^{2}\right.$, $\left.L_{\beta}^{2}\right)$.

Remark 6.2. The proof of Theorem 6.1 is based only on Lemma 6. 4. Thus Lemma 5.1 and Lemma 5.5 can be used to conclude Theorem 7.1 for $e^{t A(k)}$ and $e^{t B_{0}(k)}$. Moreover the estimates are uniform with respect to $k \in \boldsymbol{R}^{n}$. For $e^{t A(k)}$, however, there is a very simple proof, which will be presented in Lemma 8.1.

\section{§7. Estimates of $e^{t B(k)}$ near $h=0$}

The constant $C$ in Theorem 6.1 can be lound to tend to infinity as $r \rightarrow 0$. To see this more precisely, let $a>r>0$ and write (6.8) as

$$
I_{l}(t,-a, a)=I_{l}(t,-a,-r)+I_{l}(t,-r, r)+I_{l}(t, r, a) .
$$

If $\lambda \in \bar{\Sigma},|\lambda| \geq r$, then $(\lambda, k) \in \bar{\Sigma}_{r}$ for all $k \in \mathbb{R}^{n}$. Therefore (6.13), (6.14) and the argument following to (6.14) apply to the first and the last term in the right side of (7.1). Thus we have the

Lemma 7.1. For each fixed $r>0$, the limits of $I_{l}(t,-a,-r)$ and $I_{l}(t, r, a)$ exist as $a \rightarrow \infty$ in the weak topology of $\mathbb{B}\left(L_{\beta+l \delta}^{2}, L_{\beta}^{2}\right)$, and are bounded in $\mathbb{B}\left(L_{\beta+l \delta}^{2}, L_{\beta}^{2}\right)$ uniformly in $t \geq 0$ and $k \in \mathbb{R}^{n}$. 
Consequently it is the middle term of the right side of (7.1) from which the singularity arises when $k \rightarrow 0$. To derive its asymptotic behaviors, we are forced to use a $C^{\infty}$-extension of $G(\lambda, k)$ for $\operatorname{Re} \lambda<0$.

For simplifications of notations we write

$$
\begin{aligned}
& R(\lambda, k)=(\lambda-B(k))^{-1}, \quad R_{0}(\lambda, k)=\left(\lambda-B_{0}(k)\right)^{-1}, \\
& G(\lambda, k)=R_{0}(\lambda, k) P, \quad G_{1}(\lambda, k)=P R_{0}(\lambda, k) \\
& H(\lambda, k)=P R_{0}(\lambda, k) P=P G(\lambda, k)=G_{1}(\lambda, k) P,
\end{aligned}
$$

where $G(\lambda, k)$ is the same with (6.4).

We use the second resolvent equation in the form

$$
R(\lambda, k)=R_{0}(\lambda, k)+G(\lambda, k)(I-H(\lambda, k))^{-1} G_{1}(\lambda, k),
$$

which follows from (6.1) and the fact that $P$ is a projection, $P^{2}=P$. Since $R_{0}, G$ and $G_{1}$ are bounded in $\bar{\Sigma}$, the singularity arises from ( $I-$ $H(\lambda, k))^{-1}$. To study this, we need

$$
R_{0}(\lambda, k)=(\lambda-L+P)^{-1}+R_{0}(\lambda, k) A_{0}(k)(\lambda-L+P)^{-1},
$$

which is a second resolvent equation for the first equality of (5.5). This is a formal equation since $D\left(A_{0}(k)\right) \neq L_{\beta}^{2}$, but if both sides are multiplied by $P$ from the left or from the right, then the equality becomes exact, owing to nice properties of $P$ given in Lemma 4.3 (iv), and if we put

$$
D\left(\lambda, k, k^{\prime}\right)=P R_{0}(\lambda, k) A_{0}\left(k^{\prime}\right) P,
$$

then we get

$$
H(\lambda, k)=\frac{1}{\lambda+1}\{P+D(\lambda, k, k)\}
$$

since $P L=L P=0$ by Lemma 4.3 (iii).

Lemma 7.2. Put $\lambda=\boldsymbol{\gamma}+i \tau$ and identify $\Sigma=\boldsymbol{C}_{+} \times \mathbb{R}^{n}$ with $\boldsymbol{R}_{+} \times \boldsymbol{R}$ $\times \boldsymbol{R}^{n}$. Considered as functions of $\gamma, \tau$ and $k$, the operators $G(\lambda, k)$, $G_{1}(\lambda, k)$ and $H(\lambda, k)$ are in $\mathscr{B}^{\infty}\left(\bar{\Sigma} ; \boldsymbol{C}\left(L_{\beta}^{2}\right)\right)$. Also $D\left(\lambda, k, k^{\prime}\right) \in \mathscr{B}^{\infty}\left(\overline{\boldsymbol{R}}_{+}\right.$ $\left.\times \boldsymbol{R} \times \boldsymbol{R}^{n} \times \boldsymbol{R}^{n} ; \boldsymbol{C}\left(L_{\beta}^{2}\right)\right)$.

Proof. This also is due to nice properties of $P$ mentioned above. 
In fact it follows from Lemmas 4.3 (iv) and 5.5 (ii) applied to (6.10) that $\partial^{l} G / \partial \gamma^{l}, \partial^{l} G / \partial \tau^{l} \in \mathscr{B}^{0}\left(\bar{\Sigma} ; \mathbb{C}\left(L_{\beta}^{2}\right)\right)$ for all $l \in \mathbb{N}$, as a product of $P$ $\in \mathbb{C}\left(L_{\beta}^{2}, L_{\beta^{\prime}}^{2}\right)$ and $R_{0}^{l+1} \in \mathcal{B}^{0}\left(\bar{\Sigma} ; \boldsymbol{B}\left(L_{\beta^{\prime}}^{2}, L_{\beta}^{2}\right)\right)$ with $\beta^{\prime}>\beta+(l+1) \delta$. Similarly

$$
\frac{\partial}{\partial k_{j}} G(\lambda, k)=-R_{0}(\lambda, k) \frac{\partial B_{0}(k)}{\partial k_{j}} R_{0}(\lambda, k) P \in \mathscr{B}^{0}\left(\bar{\Sigma} ; \mathbb{C}\left(L_{\beta}^{2}\right)\right)
$$

since $\partial B_{0}(k) / \partial k_{j}=-i \xi_{j} \times \in \mathbb{B}\left(L_{\beta+1}^{2}, L_{\beta}^{2}\right)$ for each $\beta \in \mathbb{R}$. The proof for other derivatives of $G(\lambda, k)$, and also the proofs for $G_{1}(\lambda, k), H(\lambda, k)$ and $D\left(\lambda, k, k^{\prime}\right)$ can be carried out exactly in the same manner.

This lemma assures that the functions $G, G_{1}, H$ and $D$ possess $C^{\circ \text {. }}$. extensions for $\gamma=\operatorname{Re} \lambda \leq 0,[10]$. Denote such extensions as $\widetilde{G}(\gamma, \tau, k)$ etc. with symbol $\sim$. The extension is not unique, but any extension suffices for our purpose except for $\widetilde{H}$ which should be specified as that induced from $\widetilde{D}$ by $(7.5)$;

$$
\widetilde{H}(\gamma, \tau, k)=\frac{1}{\lambda+1}\{P+\widetilde{D}(\gamma, \tau, k, k)\} .
$$

It is evident that $\widetilde{D}$ and $\widetilde{H}$ commute with $P$ and have ranges in the space $P L_{\beta}^{2}$ of dimension $n+2$, so that they can be studied through their matrix representations for $P L_{\beta}^{2}$ with a particular choice of $\beta$. We take $\beta=0$ and choose as a basis of $P L^{2}$ the orthonormal set $\left\{\Psi_{j}\right\}_{j=-1}^{n}$ given as

$$
\Psi_{-1}=c_{1} \varphi_{0}+c_{2} \varphi_{n+1}, \quad \Psi_{j}=\varphi_{j} /\left\|\varphi_{j}\right\|_{L^{2}}, \quad 0 \leq j \leq n,
$$

where $\varphi_{j}=\varphi_{j}(\xi), 0 \leq j \leq n+1$, are those given by (4.16) and $c_{1}, c_{2}$ are so chosen that $\left\|\Psi_{-1}\right\|_{L^{2}}=1$ and $\left(\Psi_{-1}, \Psi_{n}\right)_{L^{2}}=0$. With this basis $\widetilde{D}$ has a matrix representation

$$
d\left(\gamma, \tau, k, k^{\prime}\right)=\left(d_{j l}\left(\gamma, \tau, k, k^{\prime}\right)\right),
$$

with

$$
d_{j l}\left(\gamma, \tau, k, k^{\prime}\right)=\left(\widetilde{D}\left(\gamma, \tau, k, k^{\prime}\right) \Psi_{j}, \Psi_{l}\right)_{L \varepsilon}, \quad-1 \leq j, l \leq n .
$$

It is clear from Lemma 7.2 that

$$
d_{j l}\left(\gamma, \tau, k, k^{\prime}\right) \in C^{\infty}\left(\mathbb{R} \times \mathbb{R} \times \mathbb{R}^{n} \times \mathbb{R}^{n}\right) .
$$


To investigate this matrix, we follow the argument of [4].

Lemma 7. 3. Let $e_{1}=(1,0, \cdots, 0) \in S^{n-1}$, and for $k \in \boldsymbol{R}^{n}$, set $k=\kappa \omega$, $\kappa=|k|, \omega=k / \kappa=\left(\omega_{1}, \omega_{2}, \cdots, \omega_{n}\right) \in S^{n-1}$. Then

$$
\begin{aligned}
d_{j l}(\gamma, \tau, k, k)= & \kappa d_{j l}\left(\gamma, \tau, \kappa e_{1}, e_{1}\right), \quad j, l=-1,0, \\
= & \kappa \omega_{j} d_{1 l}\left(\gamma, \tau, \kappa e_{1}, e_{1}\right), \quad 1 \leq j \leq n, \quad l=-1,0, \\
= & \kappa \omega_{l} d_{j 1}\left(\gamma, \tau, \kappa e_{1}, e_{1}\right), \quad j=-1,0,1 \leq l \leq n, \\
= & \kappa \omega_{j} \omega_{l} d_{11}\left(\gamma, \tau, \kappa e_{1}, e_{1}\right) \\
& +\kappa\left(\delta_{j l}-\omega_{j} \omega_{l}\right) d_{22}\left(\gamma, \tau, \kappa e_{1}, e_{1}\right), \quad 1 \leq j, \quad l \leq n,
\end{aligned}
$$

where $\delta_{j l}$ is Kronecker's $\delta$.

Proof. Since $A_{0}(k)=\kappa A_{0}(\omega)$, we see $d_{j l}(\gamma, \tau, k, k)=\kappa d_{j l}(\gamma, \tau, \kappa \omega, \omega)$. Let $r=\left(r_{j l}\right) \in O(n)$ with $R$ as in (4.17), and note $R A_{0}(k) R^{*}=A_{0}\left({ }^{t} r k\right)$, where ${ }^{t} r$ is the transpose of $r$. Then $\widetilde{D}\left(\gamma, \tau, k, k^{\prime}\right)=R^{*} \widetilde{D}\left(\gamma, \tau,{ }^{t} r k,{ }^{t} r k^{\prime}\right) R$ by Lemma 4.4. This can be shown first for $r \geq 0$ and then for $r \leq 0$. Thus

$$
d_{j l}\left(\gamma, \tau, k, k^{\prime}\right)=\left(\widetilde{D}\left(\gamma, \tau,{ }^{t} r k,{ }^{t} r k^{\prime}\right) R \Psi_{j}, R \Psi_{l}\right)
$$

On the other hand we have

$$
\begin{aligned}
& R \Psi_{j}=\Psi_{j}, \quad j=-1,0 \\
& R \Psi_{j}=\sum_{m=1}^{n} r_{j m} \Psi_{m}, \quad 1 \leq j \leq n
\end{aligned}
$$

Now choose $r \in O(n)$ such that $r e_{1}=\omega$ and let $j, l=-1,0$. Then ${ }^{t} r \omega=e_{1}$ and we have by $(7.9)$ and $(7.10)$,

$$
d_{j l}(\gamma, \tau, k, \omega)=\left(\widetilde{D}\left(\gamma, \tau, \kappa e_{1}, e_{1}\right) \Psi_{j}, \Psi_{l}\right)=d_{j l}\left(\gamma, \tau, \kappa e_{1}, e_{1}\right),
$$

proving the first line of the lemma. The rest of the lemma can be proved similarly by choosing suitable $r \in O(n)$, and the details will be omitted.

Define a $3 \times 3$ matrix

$$
d_{0}(\gamma, \tau, \kappa)=\left(d_{j l}\left(\gamma, \tau, \kappa e_{1}, e_{1}\right)\right), \quad j, l=-1,0,1,
$$


and a $3 \times(n+2)$ matrix

$$
\Omega_{\omega}=\left(\begin{array}{lll}
1 & 0 & 0 \\
0 & \omega & 0 \\
0 & 0 & 1
\end{array}\right)
$$

for $\omega=\left(\omega_{1}, \cdots, \omega_{n}\right) \in S^{n-1}$. Then Lemma 7.3 asserts that the $(n+2)$ $\times(n+2)$ matrix $(7.5)$ with $k=k^{\prime}=\kappa \omega$ can be written

$$
\begin{aligned}
d(\gamma, \tau, k, k)= & \kappa^{t} \Omega_{\omega} d_{0}(\gamma, \tau, \kappa) \Omega_{\omega} \\
& +\kappa\left(I_{n \div 2}-{ }^{t} \Omega_{\omega} \Omega_{\omega}\right) d_{22}\left(\gamma, \tau, \kappa e_{1}, e_{1}\right)
\end{aligned}
$$

where $I_{n+2}$ is a unit matrix of order $n+2$. It is easy to see that $\Omega_{\omega}{ }^{l} \Omega_{\omega}$ $=I_{3}$ for each $\omega \in S^{n-1}$. Therefore the decomposition $(7.12)$ is orthogonal, from which follows

$$
\sigma_{p}(d(\gamma, \tau, k, k))=\sigma_{p}\left(\kappa d_{0}(\gamma, \tau, \kappa)\right) \cup\left\{\kappa d_{22}\left(\gamma, \tau, \kappa e_{1}, e_{1}\right)\right\}
$$

Lemma 7.4. There exist a positive number $r_{1}$ and functions $\nu_{j}=\nu_{j}(\gamma, \tau, \kappa), j=1,2,3$, defined on $\Sigma_{0}^{1}=\left\{(\gamma, \tau, \kappa) \in \mathbb{R}^{3} ;|\gamma|+|\tau|<r_{1},|\kappa|\right.$ $\left\langle r_{1}\right\}$, such that

(i) $\sigma_{p}\left(d_{0}(\gamma, \tau, \kappa)\right)=\left\{\nu_{j}(\gamma, \tau, \kappa)\right\}_{j=1}^{3}$ for all $(\gamma, \tau, \kappa) \in L_{0}^{1}$,

(ii) $\nu_{j} \in C^{\infty}\left(\Sigma^{1}\right), j=1,2,3$,

(iii) $\nu_{j}(\gamma, \tau, \kappa) \neq \nu_{l}(\gamma, \tau, \kappa), j \neq l,(\gamma, \tau, \kappa) \neq(0,0,0)$,

and

(iv) if we denote by $Q_{j}(\gamma, \tau, \kappa)$ the eigen projection for $\nu_{j}(\gamma, \tau, \kappa)$ $\in \sigma_{p}\left(d_{0}(\gamma, \tau, \kappa)\right)$, then $Q_{j} \in C^{\infty}\left(\Sigma_{0}^{1}\right)$.

Proof. We see easily that

$$
d_{0}(0,0,0)=\left(\left(A_{0}\left(e_{1}\right) \Psi_{j}, \Psi_{l}\right)_{L^{2}}\right)=\left(\begin{array}{ccc}
0 & 0 & \alpha_{1} \\
0 & 0 & \alpha_{2} \\
\alpha_{1} & \alpha_{2} & 0
\end{array}\right)
$$

where $\alpha_{1}=\left(A_{0}\left(e_{1}\right) \Psi_{-1}, \Psi_{1}\right)_{L^{2}}$ and $\alpha_{2}=\left(A_{0}\left(e_{1}\right) \Psi_{0}, \Psi_{1}\right)_{L^{2}}$ are nonzero and pure imaginary. Consequently the $3 \times 3$ matrix $d_{0}(0,0,0)$ has three distinct real eigenvalues $\nu=0$ and $\nu= \pm \sqrt{-\left(\alpha_{1}^{2}+\alpha_{2}^{2}\right)} \neq 0$. This and (7.7) prove the lemma since a simple zero of a polynomial is a holomorphic function of coefficients in a small neighborhood. 
Let us turn to $\tilde{H}$ of (7.5). Let $\nu_{j}, Q_{j}$ be as above and put

$$
\begin{aligned}
& \mu_{j}(\gamma, \tau, \kappa)=\frac{1}{\gamma+i \tau+1}\left(1+\kappa \nu_{j}(\gamma, \tau, \kappa)\right), \quad j=1,2,3, \\
& \mu_{4}(\gamma, \tau, \kappa)=\frac{1}{\gamma+i \tau+1}\left(1+\kappa d_{22}\left(\gamma, \tau, \kappa e_{1}, e_{1}\right)\right)
\end{aligned}
$$

$$
\begin{aligned}
& P_{j}(\gamma, \tau, \kappa \omega)={ }^{t} \Omega_{\omega} Q_{j}(\gamma, \tau, \kappa) \Omega_{\omega}, \quad j=1,2,3, \\
& P_{4}(\gamma, \tau, \kappa \omega)=I_{n+2}-{ }^{t} \Omega_{\omega} \Omega_{\omega} .
\end{aligned}
$$

We identify the matrices $P_{j}$ with the corresponding operators in $L_{\beta}^{2}$. Combining (7.7) and (7.12) with (7.6), and using Lemma 7.4, we readily prove the

Proposition 7.1. Let $r_{1}>0$ be as in Lemma 7.4 and put $\Sigma^{1}$ $=\left\{(\gamma, \tau, k) \in \boldsymbol{R} \times \boldsymbol{R} \times \boldsymbol{R}^{n} ;|\gamma|+|\tau|<r_{1},|k|<r_{1}\right\}$. Then

(i) (a) $\sigma_{d}(\tilde{H}(\gamma, \tau, k))=\left\{\mu_{j}(\gamma, \tau,|k|)\right\}_{j=1}^{4},(\gamma, \tau, k) \in \Sigma^{1}$.

(b) $\quad \mu_{j} \in C^{\infty}\left(\Sigma^{1}\right), \quad 1 \leq j \leq 4$.

(c) $\quad \mu_{j}(0,0,0)=1, \quad 1 \leq j \leq 4$.

(d) $\frac{\partial \mu_{j}}{\partial \gamma}(0,0,0)=\frac{1}{i} \frac{\partial \mu_{j}}{\partial \tau}(0,0,0)=-1, \quad 1 \leq j \leq 4$.

(ii) (a) $P_{j}(\gamma, \tau, k) \in C^{\infty}\left(\Sigma^{1} ; C\left(L^{2}\right)\right), \quad 1 \leq j \leq 4$.

(b) $\sum_{j=1}^{4} P_{j}(0,0,0)=P$.

Using this spectral decomposition of $\tilde{H}$, we can write

$$
(I-\tilde{H}(\gamma, \tau, k))^{-1} P=\sum_{j=1}^{4}\left(1-\mu_{j}(\gamma, \tau,|k|)\right)^{-1} P_{j}(\gamma, \tau, k) .
$$

Substitution of this into (7.3) then gives

$$
R(\lambda, k)=R_{0}(\lambda, k)+\sum_{j=1}^{4}\left(1-\mu_{j}(\gamma, \tau,|k|)\right)^{-1} U_{j}(\gamma, \tau, k)
$$

where $\lambda=\gamma+i \tau \in \overline{\boldsymbol{C}}_{+}$and we have put

$$
U_{j}(\gamma, \tau, k)=\widetilde{G}(\gamma, \tau, k) P_{j}(\gamma, \tau, k) \widetilde{G}_{1}(\gamma, \tau, k),
$$

$\widetilde{G}$ and $\widetilde{G}_{1}$ being $C^{\infty}$-extensions of $G$ and $G_{1}$ of (7.2) respectively. Differ- 
entiate (7.16) with respect to $\tau$. By (6.10) and Leibniz' rule, we have for $l \in N$,

$$
\begin{aligned}
R(\lambda, k)^{l+1}= & R_{0}(\lambda, k)^{l+1} \\
& +\sum_{j=1}^{j} \sum_{m=0}^{l}\left(1-\mu_{j}(\gamma, \tau,|k|)\right)^{-m-1} U_{j, m}^{(l)}(\gamma, \tau, k) .
\end{aligned}
$$

Here $U_{j, m}^{(l)}$ are given as linear combinations of products of $\mu_{j}, U_{j}$ and their derivatives, and in particular $U_{j, l}^{(l)}=i^{-l}\left(\partial \mu_{j} / \partial \tau\right)^{l} U_{j}$. We see easily from Proposition 7.1 (i) (d) that

$$
U_{, i, l}^{(l)}(0,0,0)=P_{j}(0,0,0)
$$

and from Propositions 7.1 (i) (b) and (ii) (a) that

$$
U_{j, m}^{(l)}(\gamma, \tau, k) \in C^{\infty}\left(\bar{\Sigma}^{1} ; \mathbb{C}\left(L_{\beta}^{2}\right)\right) .
$$

Now (7.17) shows that the singularity of $R(\lambda, k)^{l+1}$ arises from the factors $\left(1-\mu_{j}(\gamma, \tau,|k|)\right)^{-m-1}$. To study this in details, consider the maps defined as

$$
h_{j}=\left(\operatorname{Re} \mu_{j}, \operatorname{Im} \mu_{j}\right) ; \Sigma_{0}^{1} \rightarrow \mathbb{R}^{2}, \quad 1 \leq j \leq 4 .
$$

Then Proposition 7.1 (i) (b) (c) (d) ensures all the conditions under which the classical implicit function theorem can be applied. Thus we have the

Proposition 7.2. There exist a positive number $r_{2}\left(\leq r_{1}\right)$ and real valued functions $\gamma_{j}(\kappa)$ and $\tau_{j}(\kappa), 1 \leq j \leq 4$, defined on the interval $I_{2}=\left(-r_{2}, r_{2}\right)$ such that for all $j$,

(i) $\gamma_{j}, \tau_{j} \in C^{\infty}\left(\bar{I}_{2}\right)$,

(ii) $\quad\left(\gamma_{j}(\kappa), \tau_{j}(\kappa), \kappa\right) \in \Sigma_{0}^{1}, \quad \kappa \in I_{2} \quad$ and

(iii) $\mu_{j}\left(\gamma_{j}(\kappa), \tau_{j}(\kappa), \kappa\right) \equiv 1, \quad \kappa \in I_{2}$.

As for asymptotic behaviors of $\gamma_{j}(\kappa)$ and $\tau_{j}(\kappa)$, we should mention the

Proposition 7.3. There exist constants $\gamma_{j}^{(0)}>0$ and $\tau_{j}^{(0)} \in \mathbb{R}$ such that when $\kappa \rightarrow 0$,

$$
\gamma_{j}(\kappa)=-\gamma_{j}^{(0)} \kappa^{2}+0\left(|\kappa|^{3}\right), \quad \tau_{j}(\kappa)=\tau_{j}^{(0)} \kappa+0\left(|\kappa|^{3}\right) .
$$


The proof can be carried out along the line given in [4], and therefore will be omitted.

We are now ready to prove the main result of this section:

Theorem 7.1. Let $\beta \geq 0$. There exist a positive number $r_{0}$ and a constant $C \geq 0$ such that for all $t \geq 0,|k| \leq r_{0}$,

$$
\begin{aligned}
\left\|e^{t B(k)} u\right\|_{L^{2}} \leq & C\left\{(1+t)^{-\beta}\left(\|u\|_{L_{\beta \delta}^{2}}+\rho_{\beta+1 / 2}(|k|)\|u\|_{L^{2}}\right)\right. \\
\left.+e^{-\eta_{0}(k)^{2} t}\|P u\|_{L^{2}}\right\} &
\end{aligned}
$$

where $\rho_{\beta}(\kappa)=1$ for $\beta<1,=\log (2+|\kappa|)$ for $\beta=1$, and $=|\kappa|^{-2(\beta-1)}$ for $\beta>1$.

Proof. We recall (6.9) and Lemma 7.1. Thus we have only to evaluate $I_{l}(t,-r, r)$ of $(7.1)$ for some sufficiently small $r>0$. Choose $r_{1}$ of Proposition 7.1 as $r$, and use $(7.17)$ to write

$$
I_{l}\left(t,-r_{1}, r_{1}\right)=I_{l}^{0}(t)+\sum_{j=1}^{4} \sum_{m=0}^{l} I_{l, j, m}(t),
$$

where

$$
I_{l}^{0}(t)=\int_{-r_{1}}^{r_{1}} e^{i \tau t} R_{0}(i \tau)^{l+1} d \tau
$$

and

$$
I_{l, j, m}(t)=\int_{-r_{1}}^{r_{1}} e^{i \tau t}\left(1-\mu_{j}(0, \tau, k)\right)^{-m-1} U_{j, m}^{(l)}(0, \tau, k) d \tau
$$

Since (6.13) and (6.14) hold with $R_{0}(\lambda)$ in place of $R(\lambda)$, we see

$$
\sup _{l \geq 0, k \in \boldsymbol{R}^{n}}\left\|I_{l}^{0}(t)\right\|_{\boldsymbol{B}\left(L_{\beta+l \delta}^{2}, L_{\beta}^{2}\right)}<+\infty 。
$$

To evaluate (7.22), we first note from Propositions 7.1 (i) (b) (d), 7.2 (iii) and 7.3 that Taylor expansion gives

$$
\begin{aligned}
\left(1-\mu_{j}(\gamma, \tau, \kappa)\right)^{-l} & =\left(\mu_{j}\left(\gamma_{j}(\kappa), \tau_{j}(\kappa), \kappa\right)-\mu_{j}(\gamma, \tau, \kappa)\right)^{-l} \\
& =\left(\lambda-\lambda_{j}(\kappa)\right)^{-l}\left\{1+O\left(|\kappa|+\left|\lambda-\lambda_{j}(\kappa)\right|\right)\right\},
\end{aligned}
$$

when $|\lambda|,|\kappa| \rightarrow 0$ where $\lambda=\gamma+i \tau$ and we defined

$$
\lambda_{j}(\kappa)=\gamma_{j}(\kappa)+i \tau_{j}(\kappa), \quad 1 \leq j \leq 4 .
$$


Also by Proposition 7.3, there exist positive constants $r_{0}\left(\leq r_{2}\right)$ and $\eta_{0}$ such that for $1 \leq j \leq 4$ and for all $|\kappa| \leq r_{0}$,

$$
\gamma_{j}(\kappa)=\operatorname{Re} \lambda_{j}(\kappa) \leq-\eta_{0} \kappa^{2}
$$

Then we get, with $\rho_{\beta}(\kappa)$ stated in the theorem,

$$
\int_{-r_{1}}^{r_{1}} \frac{d \tau}{\left|i \tau-\lambda_{j}(\kappa)\right|^{\beta}} \leq C \rho_{\beta}(\kappa)
$$

for $1 \leq j \leq 4$ and $|\kappa| \leq r_{0}$. This, together with (7.19) and (7.25), gives

$$
\begin{aligned}
\sup _{t \geq 0,|k| \leq r_{0}}\left\|I_{l, j, m}(t)\right\|_{\boldsymbol{B}\left(L_{\beta}^{2}\right)} & \leq C\left\{(1+|\kappa|) \rho_{m+1}(\kappa)+\rho_{m}(\kappa)\right\} \\
& \Xi C^{\prime} \rho_{m+1}\left(\kappa^{\circ}\right),
\end{aligned}
$$

with some constant $C \geq 0$ depending only on $l \in \mathbb{N}$.

For the case $m=l$, however, this estimate is yet unsatisfactory. An improvement is possible if we note from $(7.18),(7.19)$ and Proposition 7.3 that

(7. 28) $\left\|U_{j, l}^{(l)}(\gamma, \tau, k)-P_{j}(0,0,0)\right\|_{\boldsymbol{B}\left(L_{\beta}^{2}\right)}=O\left(|k|+\left|\lambda-\lambda_{j}(k)\right|\right)$

when $|\lambda|,|k| \rightarrow 0$. Put

$$
I_{l, j}=\int_{-r_{0}}^{r_{0}} e^{i \tau t}\left(i \tau-\lambda_{j}(k)\right)^{-l-1} d \tau P_{j}(0,0,0) .
$$

Then we get by $(7.26)$ and (7.28)

$$
\left\|I_{l, j, l}(t)-I_{l, j}\right\|_{\mathbb{B}\left(L_{\beta}^{2}\right)} \leq C\left(|\kappa| \rho_{l+1}(\kappa)+\rho_{l}(\kappa)\right) \leq C \rho_{l+1 / 2}(\kappa) .
$$

It still remains to calculate the integral in (7.29). Define $\Gamma_{1}=\{\lambda$ $\left.\in i \mathbb{R} ;|\operatorname{Im} \lambda| \leq r_{1}\right\}, \Gamma_{2}=\left\{\lambda \in C_{-} ;|\lambda|=r_{1}\right\}$ and put $\Gamma_{3}=\Gamma_{1} \cup \Gamma_{2}$. Put

$$
J_{j, m}^{(l)}=\int_{\Gamma_{m}} c^{\lambda t}\left(\lambda-\lambda_{j}(k)\right)^{-(l+1)} d \lambda, l \in \mathbb{N}, 1 \leq j \leq 4,1 \leq m \leq 3
$$

Since $\Gamma_{3}$ is a simple closed curve and since $\lambda_{j}(k)$ are inside of $\Gamma_{3}$ for $|\kappa| \leq r_{0}$, a residue calculus yields, if a positive direction is taken for $\Gamma_{3}$,

$$
J_{j, 3}^{(l)}=\frac{2 \pi}{l !} t^{l} e^{\lambda_{j}(k) t}
$$

On the other hand, $\left|\lambda-\lambda_{j}(\kappa)\right|$ is strictly bounded away from zero for all $\lambda \in \Gamma_{2}$ and $|\kappa| \leq r_{0}$. Thus we find easily

$$
\left|J_{j, 2}^{(l)}\right| \leq C(1+t)^{-1}
$$


where $C \geq 0$ does not depend on $t \geq 0$ and $|\kappa| \leq r_{0}$. Clearly the integral in (7.29) is equal to $i J_{j, 1}^{(l)}=i\left(J_{j, 3}^{(l)}-J_{j, 2}^{(l)}\right)$, and so, substituting (7.31) and (7.32) into (7.29), and then combining this with (7.30), we get

$$
\begin{array}{r}
\left\|I_{l, j, l}(t)-\frac{t^{l}}{l !} e^{\lambda_{j}(\kappa) t} P_{j}(0,0,0)\right\|_{\boldsymbol{B}\left(L_{\beta}^{2}\right)} \\
\leq C\left\{(1+t)^{-1}+\rho_{l+1 / 2}(\kappa)\right\} .
\end{array}
$$

Substitute (7.23), (7.27) and (7.33) into (7.20), and use Proposition 7.1 (ii) (b) and (7.25) to get

$$
\begin{aligned}
\left\|I_{l}\left(t,-r_{1}, r_{1}\right) u\right\|_{L_{\beta}^{2}} \leq & C\left\{\|u\|_{L_{\beta+l o}^{2}}+t^{l} e^{-\eta_{0} \kappa z t}\|P u\|_{L^{2}}\right. \\
& \left.+\left((1+t)^{-1}+\rho_{l+1 / 2}(\kappa)\right)\|u\|_{L^{2}}\right\} .
\end{aligned}
$$

This and Lemma 7.1 with $r=r_{1}$, substituted into (7.1) and then into (6.9), prove the theorem for the case $\beta \in N$. For general $\beta \geq 0$, it suffices to use an interpolation.

\section{§ 8. Decay Estimates of $e^{t B}$}

The estimates given in Theorems 6.1 and 7.1 enable us to derive decay estimates of $e^{i B}$ in the space $H_{l, 0,2}$ of (2.6). The estimates in the space $\dot{H}_{l, \beta}$ which are necessary in the following section can then be obtained by the aid of nice regularity properties of $K$ stated in Lemma 4.2.

To accomplish this, we first consider $B$ of (3.7) in $H_{l, \beta, p}$ with domain (3.8) with $\dot{H}_{l, \beta}$ replaced by $H_{l, \beta, p}$. Then $B$ is a generator for any $l \in \boldsymbol{R}, \beta \geq 0$ and $1 \leq p<\infty$, which can be shown just in the same way as Theorem 3.1. Moreover, using arguments for Lemma 6.1, we can prove that $e^{t B}$ is a contraction semigroup in $H_{l, 0,2}$ since $L$ is nonpositive there by Lemma 4.3 (i).

We now state decay estimates of $e^{t B}$ in $H_{l, 0,2}$.

Theorem 8. 1. Let $l \in N$ and $p \in[1,2]$. For $\beta \in\left[0, \frac{n}{2}\left(\frac{1}{p}-\frac{1}{2}\right)\right.$ $\left.+\frac{1}{2}\right)$, there exists a constant $C \geq 0$ such that

$$
\begin{aligned}
\left\|e^{t B} u\right\|_{l, 0,2} \leq & C\left\{(1+t)^{-\beta}\left(\|u\|_{l, \beta \delta, 2}+\|u\|_{L^{p, 2}}\right)\right. \\
& +(1+t)^{-(n / 2)(1 / p-1 / 2)}\|P u\|_{\left.L^{p, 2}\right\}}
\end{aligned}
$$


holds for all $t \geq 0$ and $u \in H_{l, \beta \delta, 2} \cap L^{p, 2}$, where $\delta \geq 0$ is that of [Q] (ii) and $L^{p, 2}$ was defined in (2.13).

Proof. $B$ is related to $B(k)$ through (5.1) and (5.2). Then

$$
\left\|e^{t B} u\right\|_{l, 0,2}^{2}=\int_{\mathbb{R}^{n}}(1+|k|)^{2 l}\left\|e^{t B(k)} \widehat{u}(k, \cdot)\right\|_{L^{2}\left(\boldsymbol{R}_{\xi}^{n}\right)}^{2} d k
$$

by the definition (2.7). Let $r_{0}>0$ be as in Theorem 7.1 and divide the region of integration in the above into two regions $|k| \geq r_{0}$ and $|k| \leq r_{0}$, Denote the respective integrals as $I_{1}$ and $I_{2}$. If we take $r=r_{0}$, then Theorem 6.1 gives

$$
I_{1} \leq C(1+t)^{-2 \beta}\|u\|_{i, \beta \delta, 2}^{2},
$$

for any $\beta \geq 0$, while by Theorem 7.1 and Hölder

$$
\begin{aligned}
I_{2} \leq C\left[( 1 + t ) ^ { - 2 \beta } \left\{\|u\|_{l, \beta \delta, 2}^{2}\right.\right. & \left.+\left(\int_{|k| \leq r_{0}} \rho_{\beta+1 / 2}(|k|)^{2 q^{\prime}} d k\right)^{1 / q^{\prime}}\|\hat{u}\|_{Y_{q}}^{2}\right\} \\
+ & \left.\left(\int_{|k| \leq r_{0}} e^{-2 q^{\prime} \eta_{0}\left|k^{\prime}\right|^{2 t}} d k\right)^{1 / q^{\prime}}\|P \hat{u}\|_{Y_{q}}^{2}\right],
\end{aligned}
$$

where $Y_{q}=L^{2 q}\left(\boldsymbol{R}_{k}^{n} ; L^{2}\left(\boldsymbol{R}_{\xi}^{n}\right)\right)$ and $1 / q+1 / q^{\prime}=1, q \geq 1$. Put $p=2 q /(2 q-1)$ $=2 q^{\prime} /\left(2 q^{\prime}+1\right)$. Then $1 \leq p \leq 2$, and

$$
\int_{\left|k^{\prime}\right| \leq r_{0}} \rho_{\beta+1 / 2}(|k|)^{2 q^{\prime}} d k<+\infty
$$

if $\beta<\frac{n}{4 q^{\prime}}+\frac{1}{2}=\frac{n}{2}\left(\frac{1}{p}-\frac{1}{2}\right)+\frac{1}{2}$, while

$$
\left(\int_{|k| \leq r_{u}} e^{-2 q^{\prime} \eta_{0}|k|^{2} t} d k\right)^{1 / q^{\prime}} \leq C(1+t)^{-\left(n / 2 q^{\prime}\right)}=C(1+t)^{-n(1 / p-1 / 2)}
$$

and

$$
\|\widehat{\imath}\|_{Y_{q}} \leq C\|u\|_{L^{p, 2}} .
$$

Now the theorem follows immediately from these.

In the space $\dot{H}_{l, \beta}$ defined by (2.8), $e^{t B}$ has decay estimates slightly weaker than those in the above.

Theorem 3.2. Let $\delta \in[0,1)$ be as in [Q] (ii), and $p \in[1,2]$. 
For any $\alpha \geq 0$ such that $\alpha \leq \frac{n}{2}\left(\frac{1}{p}-\frac{1}{2}\right), \alpha<\frac{1}{\delta}$, and for all $l \in \boldsymbol{R}$ and $\beta>n / 2$, there exists a constant $C \geq 0$ such that

$$
\left\|e^{t B} u\right\|_{l, \beta} \leq C(1+t)^{-\alpha}\left(\|u\|_{l, \beta+\alpha \delta}+\|u\|_{L^{p, 2}}\right)
$$

for any $u \in \dot{H}_{l, \beta+\alpha \delta} \cap L^{p, 2}$. Moreover if $u$ is such that $P u=0$, then we can take $\alpha \in\left[0, \min \left(\frac{n}{2}\left(\frac{1}{p}-\frac{1}{2}\right)+\frac{1}{2}, \frac{1}{\delta}\right)\right)$.

To prove this theorem, we first recall $A_{0}$ of (3.9) and $\Lambda$ of (4.1), and define

$$
A=A_{0}+\Lambda, \quad D(A)=D\left(A_{0}\right) .
$$

It is easily found that the proof of Theorem 3.1 applies also to $A$ to conclude that $A$ with a maximal domain $D(A)=D(B)$ is a semigroup generator in $H_{l, \beta, p}$ as well as in $\dot{H}_{l, \beta} . \quad e^{t A}$ has estimates similar to those in Theorem 6.1 (cf. Remark 6.2), namely,

Lemma 8. 1. Let $l \in \boldsymbol{R}, \alpha \geq 0, \beta \geq 0$ and $p \in[1, \infty)$. Then

$$
\left\|e^{t A}\right\|_{B(X, Y)} \leq C(1+t)^{-\alpha}, \quad t \geq 0
$$

for either pair $(X, Y)$ of $\left(\dot{H}_{l, \beta+\alpha \hat{\delta}}, \dot{H}_{l, \beta}\right)$ and $\left(H_{l, \beta+\alpha \delta, p}, H_{l, \beta, p}\right)$.

Proof. Using (3.11), we see $\left(e^{t A} u\right)^{\wedge}(k, \xi)=e^{-t(i\langle k, \xi\rangle+\nu(\xi))} \hat{u}(k, \xi)$. Since $\sup _{x \geq 0} x^{\alpha} e^{-t x} \leq c t^{-\alpha}$ for $t, \alpha \geq 0$, the lemma follows from (4.3).

Proof of Theorem 8.2. First note that if $\gamma \geq \alpha \geq 0$, and $\beta=$ $\min (\alpha, \gamma+\alpha-1)$, then

$$
\int_{0}^{\imath}(1+t-s)^{-r}(1+s)^{-\alpha} d s \leq C(1+t)^{-\beta} \times \begin{cases}\log (2+t), & \gamma=1 \\ 1, & \gamma \neq 1,\end{cases}
$$

holds for all $t \geq 0$.

Further, for $v=v(t) \in C^{0}([0, \infty) ; X)$ with a Banach space $X$, and for $\mu \in \boldsymbol{R}$, we define

$$
\|v\|_{X, \mu}=\sup _{t \geq 0}(1+t)^{\mu}\|v(t)\|_{X} .
$$

We have already noted that $B$ is a generator in both $H_{l, \beta, p}$ and 
$\dot{H}_{l, \beta}$. Write $v(t)=e^{t B} u_{0}$. Since $B=A+K$ with $D(B)=D(A)$ where $K$ is defined in (4.2) or (4.11), then $v(t)$ satisfies

$$
v(t)=e^{t A} u_{0}+\int_{0}^{t} e^{(t-s) A} K v(s) d s,
$$

for all $t \geq 0$.

Let $\gamma_{0} \in(1,1 / \delta)$ and $\alpha \in\left[0, \gamma_{0}\right]$. Since $\gamma_{0}>1, \min \left(\alpha, \gamma_{0}+\alpha-1\right)=\alpha$. Then, applying Lemma 8.1 and (8.2) to (8.4), we get

$$
\begin{aligned}
\|v(t)\|_{l, \beta} \leq & C\left\{(1+t)^{-u}\left\|u_{0}\right\|_{l, \beta+a \delta}\right. \\
& \left.+\int_{0}^{t}(1+t-s)^{-r_{0}} \times\|K v(s)\|_{l, \beta+\gamma_{0} \delta} d s\right\} \\
\leq & C(1+t)^{-\alpha}\left(\left\|u_{0}\right\|_{l, \beta+\alpha \delta}+\|K v\|_{\dot{H}_{l, \beta+\gamma_{0} \delta}, \alpha}\right) .
\end{aligned}
$$

But $\|K v(s)\|_{l, \beta+\gamma_{0} \delta} \leq C\|v(s)\|_{l, \beta+\gamma_{0} \delta-1}$ in view of Lemma 4.2 (i) (c), so that (8.5) indicates that

$$
\|v\|_{X, \alpha} \leq C\left(\left\|u_{0}\right\|_{Y}+\|v\|_{Z, \alpha}\right)
$$

holds for

$$
X=\dot{H}_{l, \beta}, \quad Y=\dot{H}_{l, \beta+\alpha \delta}, \quad Z=\dot{H}_{l, \beta+\gamma_{0} \delta-1} .
$$

Since $\gamma_{0} \delta-1<0$ by our choice of $\gamma_{0}$, we can iterate (8.5) to verify that (8.6) is true eventually for

$$
X=\dot{H}_{l, \beta}, \quad Y=\dot{H}_{l, \beta+\alpha \delta}, Z=\dot{H}_{l, 0}
$$

where use was made of $(2.12)$.

Choose a $p_{0} \geq 2$ such that $\max \left(n / 2,1 /\left(1-\gamma_{0} \delta\right)\right)<p_{0}<+\infty$ if $n \geq 3$ and $\max \left(2,1 /\left(1-\gamma_{0} \delta\right)\right)<p_{0}<\infty$ if $n=2$. Put $\beta=0$ in (8.4) and use Lemma 4.2 (i) (e) with $\beta=0, \gamma=\gamma_{0} \delta, p=p_{0}$ and we find that (8.6) is true also for the case

$$
X=\dot{H}_{l, 0}, \quad Y=\dot{H}_{l, \alpha \delta}, \quad Z=\dot{H}_{l, 0, p_{0}} .
$$

In a similar way, Lemmas 4.2 (i) (d), 8.1 and (8.2), applied to (8.4), give rise to (8.6) for $X=H_{l, 0, p_{2}}, Y=H_{l, \alpha \delta, p_{2}}$ and $Z=H_{l, 0, p_{1}}$ for any $1<p_{1}, p_{2} \leq p_{0}, 1 / p_{1}-1 / p_{2} \leq 1 / p_{0}$. Since $1 / p_{0}>0$, this result can be iterated to conclude again (8.6) for the case

$$
X=H_{l, 0, p_{0}}, \quad Y=H_{l, \alpha \delta, p_{0}}, \quad Z=H_{l, 0,2} .
$$


If $\beta>n / 2$ and $p \geq 2$, then $\dot{H}_{l, \beta+\alpha \delta}, \subset H_{l, \alpha \delta, p}$ with

$$
\|u\|_{l, \alpha \delta, p} \leq C\|u\|_{l, \beta+\alpha \delta} .
$$

Hence (8.7), (8.8) and (8.9) can be combined to obtain (8.6) for

$$
Y=\dot{H}_{l, \beta}, \quad Y=\dot{H}_{l, \beta+\alpha \hat{o}}, \quad Z=\dot{H}_{l, 0,2} .
$$

On the other hand, if we choose $\alpha \leq \frac{n}{2}\left(\frac{1}{p}-\frac{1}{2}\right)$, or $\alpha<\frac{n}{2}\left(\frac{1}{p}-\frac{1}{2}\right.$ $\left.+\frac{1}{n}\right)$ provided $P u=0$, then Theorem 8.1 yields

$$
\|v\|_{H_{l, 0,2}, \alpha} \leq C\left(\|u\|_{l, \alpha \delta, 2}+\|u\|_{L^{p, 2}}\right) .
$$

Substitute this into (8.6) with $(X, Y, Z)$ specified by $(8.11)$, and use (8.10) to complete the proof of the theorem.

\section{§ 9. Existence of Solutions in the Large in Time}

The decay estimates derived for the linear semigroup $e^{t B}$ in Theorem 8.2 are sufficient to ensure solutions in the large in time to our nonlinear problem (3.12). To be precise, we can now prove the main result of this paper, namely,

Theorem 9. 1. Let $n \geq 2$, Suppose [Q] of Section 1 be fulfilled. Let $\gg \frac{n}{2}, \beta>\frac{n}{2}-\delta$ and $p \in[1,2)$, and put $\alpha=\min \left(\frac{n}{2}\left(\frac{1}{p}-\frac{1}{2}\right), 1\right)$. Then there exist positive constants $a_{0}, a_{1}$, and for any initial $u_{0}$ in $\dot{H}_{l, \beta+\alpha \delta} \cap L^{p, 2}$ with $\left\|u_{0}\right\|_{l, \beta+\alpha \delta}+\left\|u_{0}\right\|_{L^{p, 2}} \leq a_{0}$, (3.13) possesses a unique solution $u=u(t)$ in $C^{0}\left([0, \infty) ; \dot{H}_{l, \beta}\right)$ satisfying.

$$
\|u(t)\|_{l, \beta} \leq a_{1}(1+t)^{-\alpha}
$$

for all $t \geq 0$. Here $\delta \in[0.1$ ) is as in [Q] (ii).

Remark 9.1. The optimal decay order of (9.1) is $\alpha=n / 4$ with $p=1$ when $n=2,3,4$, and $\alpha=1$ with $p \in[1,2 n /(n+4)]$ when $n \geq 5$. On the other hand no solutions have been found in the large in time for the case $p=2$ for which $\alpha=0$, or what is the same thing, for initials $u_{0}$ belonging only to $\dot{H}_{l, \beta}$. Recall that for the case of cutoff hard potentials, the optimal decay order is $\alpha=n / 4$ for all $n \geq 2$, and moreover that solu- 
tions exist in the large in time for $p=2,[9],[13]$.

Remark 9.2. Theorem 3.2 (b) states that $u=u(t)$ of Theorem 9.1 is a unique solution to $(3.12)$ in $\dot{H}_{l-1, \beta-1}$ in $[0, \infty)$. Consequently (9.1) indicates that the solutions $f=g+g^{1 / 2} u$ to (1.1) tends to the Maxwellian $g$ with order $(1+t)^{-\alpha}$ when $t \rightarrow \infty$. Physically this means that the gas is stable for a small disturbance to an equilibrium.

Proof of Theorem 9.1. First we shall prove that

$$
\|\Gamma[u, v]\|_{L 1,2} \leq C\|u\|_{l, \beta}\|v\|_{l, \beta}
$$

holds for all $l \geq 0$ and $\beta>n / 2-\delta$. For this, we put $u_{0}(\xi)=\|u(\cdot, \xi)\|_{L^{2}\left(\boldsymbol{R}_{x}^{n}\right)}$ and obtain by Schwarz

$$
\left\|\Gamma_{j}[u, v](\cdot, \xi)\right\|_{L^{1}\left(\boldsymbol{R}_{x}^{n}\right)} \leq \Gamma_{j}\left[u_{0}, v_{0}\right](\xi)
$$

where $\Gamma_{j}, j=1,2$ are defined in (3.4). The $L^{2}\left(\mathbb{R}_{\xi}^{n}\right)$-norm of the right side is majorized by its $\dot{H}_{l, \beta+\delta}$-norm since $\beta+\delta>n / 2$. Use first Lemma 3.1 and (3.5) to obtain (9.2) for $l=0$ and then (2.12) for all $l \geq 0$.

Let $\gamma_{0}=1$ for $n \geq 3$ and choose a $\gamma_{0}$ such that $1-\alpha<\gamma_{0}<1$ if $n=2$, where $\alpha$ is the same as in the statement of the theorem. Note that

$$
r_{0} \in[0, \min ((n+2) / 2,1 / \delta))
$$

and moreover that for $\alpha \in(0,1)$,

$$
\min \left(\gamma_{0}, 2 \alpha, \gamma_{0}+2 \alpha-1\right)>\alpha .
$$

Define the bilinear operator $N$ as

$$
N[u, v](t)=\int_{0}^{t} e^{(t-s) B} \Gamma[u(s) v(s)] d s .
$$

Let $X=C^{0}\left([0, \infty) ; \dot{H}_{l, \beta}\right)$. By virtue of Corollary 3.1 and Theorem 3. $1, N$ is a continuous map from $X \times X$ into $X$. Noting Lemma 4.5 and (9.3), we apply Theorem 8.2 to $(9.5)$. Then

$$
\begin{gathered}
\|N[u, v](t)\|_{l, \beta} \leq C \int_{0}^{t}(1+t-s)^{-r_{0}}\left\{\|\Gamma[u(s), v(s)]\|_{l, \beta+r_{0} \delta}\right. \\
+\|\Gamma[u(s), v(s)]\|_{\left.L^{1,2}\right\}} d s .
\end{gathered}
$$

By the aid of Lemma 3.1 (i) and (9.2), we can majorize the right 
side by

$$
C \int_{0}^{t}(1+t-s)^{-r_{0}}\|u(s)\|_{l, \beta}\|v(s)\|_{l, \beta} d s .
$$

Here the fact $\gamma_{0} \leq 1$ is essential in order that we can apply Lemma 3.1. Recalling the definition (8.3), we put

$$
\|\cdot\| \cdot\|=\| \cdot \|_{\dot{H}_{l, \beta, \alpha}} \cdot
$$

In view of (8.2) and (9.5), (9.6) is majorized by $C(1+t)^{-\alpha}\|\imath \imath\|\|v\| \|$. Thus we have proved

$$
\|N[u, v]\| \leq C\|u\|\|v\| \| .
$$

For each fixed $u_{0} \in \dot{H}_{l, \beta} \cap L^{p, 2}$, define the map $H$ as

$$
H[u](t)=e^{t B} u_{0}+N[u](t)
$$

which maps $X$ into itself continuously. We evaluate $\left\|e^{t B} u_{0}\right\|$ by Theorem 8. 1. This and (9.6) give

$$
\|H[u]\| \leq C_{0}\left(\left\|u_{0}\right\|_{l, \beta+\alpha \delta}+\left\|u_{0}\right\|_{L^{p, 2}}\right)+C_{1}\|u\|^{2} .
$$

Note that $C_{0} \geq 1$ since $e^{t B}$ is a semigroup. Also by (9.7) we have

$$
\begin{aligned}
\|H[u]-H[v]\| & =\|N[u-v, u+v]\| \\
& \leq C_{1}(\|u\|+\|v\|)\|u-v\| .
\end{aligned}
$$

Choose a $\mu \in(0,1)$, and put $a_{0}=\mu^{2} / 4 C_{0} C_{1}$ and $a_{1}=\mu / 2 C_{1}$. Then if $\left\|u_{0}\right\|_{l, \beta+\alpha \delta}$ $+\left\|u_{0}\right\|_{L^{p, 2}} \leq a_{0}$ and if $\|u\|_{\alpha},\|v\|_{\alpha} \leq a_{1}$, it follows from (9.8) and (9.9) that

$$
\|H[u]\| \leq \mu a_{1},\|H[u]-H[v]\| \leq \mu\|u-v\| .
$$

This states that $H$ is a contraction map on the complete metric space $V=\left\{u \in C^{0}\left([0, \infty) ; \dot{H}_{l, \beta}\right) ;\|u\| \leq a_{1}\right\}$ with the metric $d(u, v)=\|u-v\|$. Hence $H$ has a unique fixed point $u=H[u]$ in $V$. This $u$ is, of course, a solution to (3.13) satisfying (9.1). Moreover it is unique not only in $V$ but also in $X=C^{0}\left([0, \infty) ; \dot{H}_{l, \beta}\right)$. For, if there is a solution $v$ $=v(t) \in X, v=H[v]$, other than the solution $u$ found above, then $v \notin V$ because of the uniqueness in $V$, and hence it follows from the continuity of $v(t)$ in $t$ and the fact $\|v(0)\|_{l, \beta}=\left\|u_{0}\right\|_{l, \beta} \leq a_{0}=\left(\mu / 2 C_{0}\right) a_{1}<a_{1}$ that there is a $T_{0}>0$ satisfying

$$
\|v\|_{T_{0}} \equiv \sup _{0 \leq t \leq T_{0}}(1+t)^{\alpha}\|v(t)\|_{l, \beta} \leq a_{1},
$$


and

$$
\left(1+T_{0}\right)^{u}\left\|v\left(T_{0}\right)\right\|_{l, \beta}-a_{1} .
$$

On the other hand it is easy to check that (9.10) remains valid if the norm $\|\cdot\|$ is replaced by the norm $\|\cdot\|_{T}$ of $(9.11)$ for any $T>0$. Hence $\|v\|_{T_{0}}=\|H[v]\|_{T_{0}} \leq \mu a_{1}<a_{1}$. This contradicts to (9.12). Thus there is no such $a v$ and $u$ is unique in $X$.

\section{Refereneers}

[1] Asano, K., On the initial boundary value problem of the nonlinear Boltzmann equation in an exterior domain, to appear.

[2] Caflish, E., The Boltzmann equation with a soft potential, Parts I \& II, Commun. Math. Phys., 74 (1980), 71-95, 97-109.

[3] Carleman, T., Problèmes Mathematiques dans la Théorie Cinétique des Gaz, Almquist et Wiksell, Uppsala, 1957.

[4] Ellis, R. S. and Pinsky, M. A., The first and second fluid approximations to the linearised Boltzmann equation, J. Math. pures et appl., 54 (1975), 125-156.

[5] Guiraud, J. P., An $H$ theorem for a gaz of rigid spheres in a bounded domain, Théories Cinétiques Classiques et Relativistés, C.N.R.S., Paris, 1975.

[6] Grad, H., Asymptotic theory of the Boltzmann equation, Rarefied Gas Dynamics, 1 (Laurmann, J. A., Ed.), Academic Press, New York, 1963.

[7] - Asymptotic equivalence of the Navier-Stokes and nonlinear Boltzmann equation, Proc. Symp. Appl. Math., 17 (Finn, R., Ed.) 154-183, Amer. Math. Soc., Providence, 1965.

[8] Kato, T., Perturbation Theory of Linear Operators, 1st ed., Springer, New York, 1966.

[9] Nishida, T. and Inai, $K$.. Global solutions to the initial value problem tor the nonlinear Boltzmann equation, Publ. RIMS, Kyoto Univ., 12 (1976), 229-239.

[10] Seely, R. T., Extension of $C^{\infty}$ functions defined in a half space, Proc. A.M.S., 15 (1964), 625-626.

[11] Shizuta, Y. (private communication)

[12] Shizuta, Y. and Asano, K., Global solutions of the nonlinear Boltzmann equation in a bounded domain, Proc. Japan Acad., 53 Ser. A (1977), 3-5.

[13] Ukai, S., On the existence of global solutions of mixed problem for the nonlinear Boltzmann Equation, Proc. Japan Acad., 50 (1974), 179-184.

[14] Les solutions globales de l'équation de Boltzmann dans l'espace tout entier et dans le demi-espace, C. R. Acad. Sc., Paris, 282A (1976), 317-320.

[15] Ukai, S. and Asano, K., On the initial boundary value problem of the linearized Boltzmann equation in an exterior domain, Proc. Japan Acad., 56 Ser. A (1980), $12-17$. 
